\title{
Short-range three-nucleon forces and low-energy constants
}

\author{
J. Adam, Jr., ${ }^{1}$ M.T. Peña, ${ }^{2}$ and A. Stadler ${ }^{3,4}$ \\ 1 Nuclear Physics Institute, Řežnear Prague, CZ-25068, Czech Republic \\ ${ }^{2}$ Instituto Superior Técnico, Centro de Física das Interacções Fundamentais, \\ and Department of Physics, Av. Rovisco Pais, P-1049-001 Lisboa, Portugal \\ 3 Centro de Física Nuclear da Universidade de Lisboa, \\ Av. Gama Pinto 2, P-1649-003 Lisboa, Portugal \\ 4 Departamento de Física, Universidade de Évora, \\ Colégio Luís Verney, P-7000-671 Évora, Portugal
}

(Dated: February 7, 2019)

\begin{abstract}
The $3 N$ forces due to $\pi-\rho, \pi-\sigma$ and $\pi-\omega$ exchanges following from the nucleon Born diagrams and diagrams with an intermediate $N^{*}(1440)$ are re-analyzed. The cancellation between $\pi-\sigma$ and $\pi-\omega$ forces is rather sensitive to the values of the coupling constants and to the form of the $\pi N N$ vertex. Experimental uncertainties in the parameters of the TM $\pi-\pi$ potential are assessed. They lead to uncertainties in theoretical predictions of the triton binding energy of about $\pm 0.4 \mathrm{MeV}$. The low-energy limit of $\pi-\sigma$ and $\pi-\omega$ potentials is performed. It defines the coupling constants of effective contact $\pi N N N N$ vertices, which are compared with the corresponding contact vertices of Chiral Perturbation Theory.

PACS numbers: 21.30.-x, 21.30.Fe, 21.45.+v, 21.10.-k
\end{abstract}




\section{INTRODUCTION}

Nucleon-nucleon $(N N)$ and three-nucleon $(3 N)$ potentials to be used in calculations of properties of few nucleon systems at low and intermediate energies are these days successfully modeled through meson exchanges. Modern $N N$ potentials based on this picture provide an impressive description (with $\chi^{2} /$ datum $\sim 1$ ) of the $N N$ scattering data below the pion production threshold and of the deuteron properties [1, 2]. These potentials often employ the underlying meson-nucleon dynamics only in one-boson-exchange approximation. One reason for this is clearly the desire for a relatively simple formalism, but it was also supported by extensive studies of the Bonn group [3] which showed numerous cancellations between classes of diagrams with two and more exchanged mesons. The meson-exchange approach with heavy meson (and nucleon) resonances has been supplemented recently by a model-independent construction from a low-energy realization of QCD: chiral perturbation theory (ChPT). While some theoretical uncertainties in the formulation of this theory persist [4], the construction of the $N N$ potential in this framework is already approaching maturity [5, 6, 7] with the next-to-next-to leading order (NNLO) potential also describing the $N N$ data fairly well. Moreover, a recent paper [8] by the Jülich-Bochum group provides a detailed comparison of ChPT with phenomenological potentials based on heavy meson exchange, and shows that the low-energy constants of ChPT appearing in the two-nucleon sector of the chiral Lagrangian agree reasonably well with those following from reducing the heavy meson operators to their contact form. Therefore a mutual positive influence between the two approaches should be expected for the future: the phenomenological potentials should provide a hint on the values of the chiral low-energy constants (which are not always easily obtained from the experiment) and the chiral constraints should be imposed on the phenomenological models at low energies. In this spirit some studies of ChPT $3 N$ force were performed [9, 10], although more extensive numerical calculations are still needed. To describe the data one might have to consider the chiral $3 N$ force from the next order of the chiral expansion [10].

In the present paper we investigate the role of some short-range heavy meson exchanges in the $3 N$ potential with respect to the binding energy of the $3 N$ system. In addition to the dominant $\pi-\pi$ component of the $3 N$ potential [11, 12], we include the force following from $\pi-\rho$ exchange [13] and the terms involving $\pi-\omega$ or $\pi-\sigma$ exchanges from Ref. [14]. The 

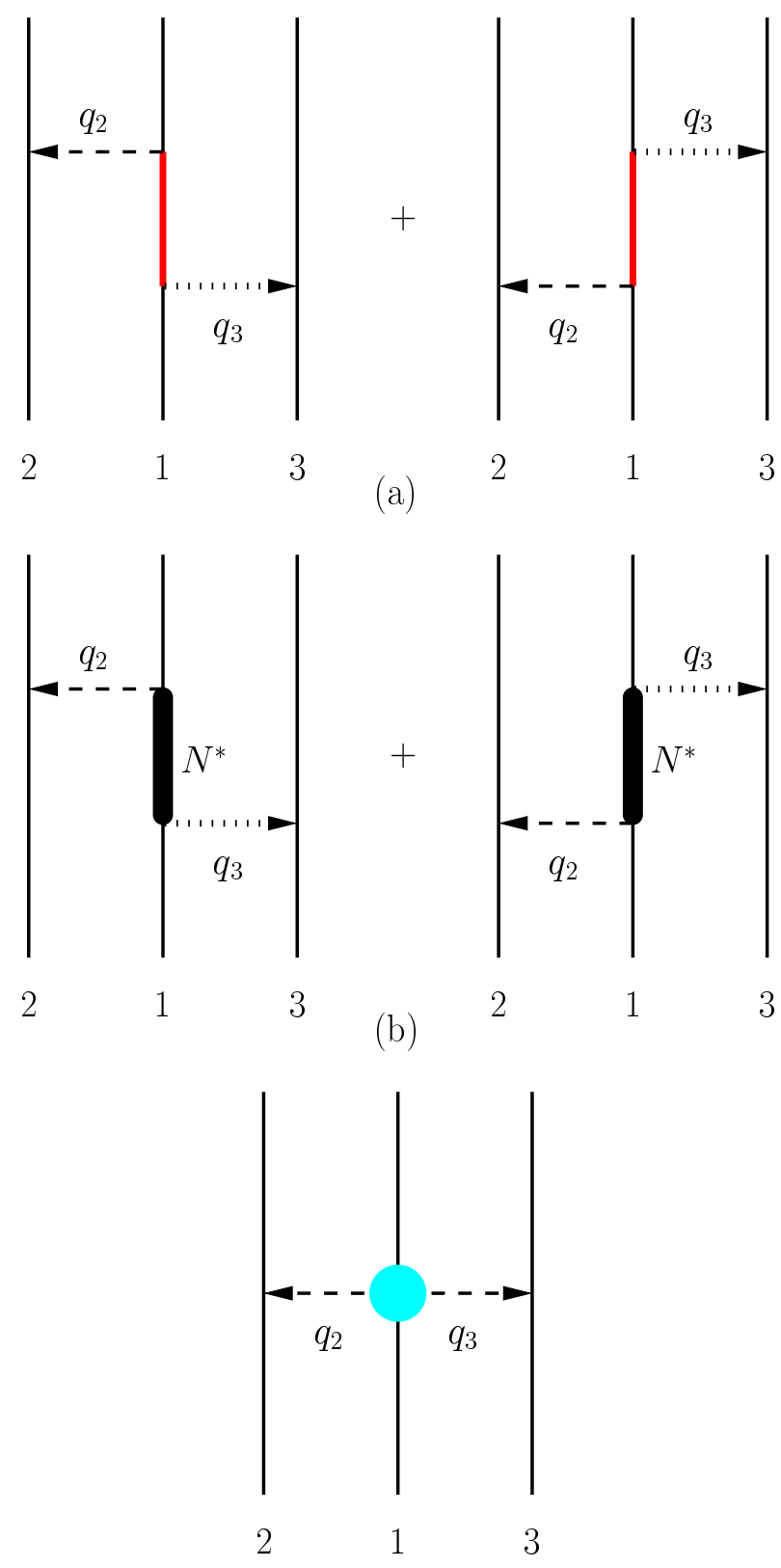

(c)

FIG. 1: Contributions to the $3 N$ force. Diagrams for (a) nucleon Born contributions, (b) intermediate Roper resonance, and (c) $\pi$ re-scattering. Diagrams (a) and (b) involve one pion and one heavy-meson exchange. They are called "long-short range $3 N$ forces". From diagrams (a), iterations of the $N N$ potential are removed, as described in Appendix A Diagram (c) involves only pion exchanges. It is called a "long-long range $3 N$ force". The meson momenta are defined as $\mathbf{q}_{i}=\mathbf{p}_{i}^{\prime}-\mathbf{p}_{i}, i=1,2,3$. 
latter potentials were obtained both from diagrams with the Roper resonance $N^{*}(1440)$ (Fig. 1b) and from the nucleon Born terms (often imprecisely called pair- or Z-diagrams) where an off-mass-shell nucleon propagates in the intermediate state (Fig. 1a). All contributions of the Born diagrams are of relativistic order. The parts with negative energy propagation, being genuine "Z-diagram" contributions, are included in the irreducible $3 N$ potential. Also the part with off-shell nucleons propagating with positive energy cannot be omitted. These terms differ in general from the iteration of the $N N$ potential (which is not to be included in the $3 N$ force and has to be subtracted from the total amplitude explicitly) and thus also contribute to the $3 N$ potential.

The purpose of this paper is to study the effects of the $3 N$ forces based on pion-pion and pion-heavy-meson exchanges. It is discussed how strongly they depend on experimental uncertainties in the determination of the phenomenological constants in terms of which these potentials are parameterized, and on the model dependence of the nucleon Born contributions. These inherent uncertainties should provide - for such semi-phenomenological meson exchange models - theoretical error bars of the calculated bound state energy of the trinucleon system.

The latest version of the phenomenological Tucson-Melbourne (TM) potential [11, 12] $\pi-\pi$ potential is parameterized in terms of three constants $a, b, d$. These constants are determined from $\pi N$ scattering data and we look at the variations of the triton binding energy within the range of their experimental uncertainty, complementing thereby the usually studied sensitivity to the variation of the form factor cut-off parameter.

The pion-heavy-meson exchanges contributions contain also some not very well determined constants, in particular the coupling constants in vertices involving the Roper resonance $N^{*}$. But besides that the Born terms also depend on the employed form of the $\pi N N$ couplings. It is often assumed that the pseudovector (PV) coupling is preferable: this is based on the experience with modeling relativistic OPE $N N$ potentials where the pseudoscalar (PS) $\pi N N$ coupling implies unrealistic enhancement of the intermediate negative energy states. It is also claimed that a PV coupling is preferred because it does not require the non-minimal contact terms to conform with chiral symmetry in processes like pion electroproduction on the nucleon.

However, in constructing the $\pi N$ scattering amplitude, or amplitudes of heavy meson production in pion absorption on the nucleon, one has to be more careful. In these cases one 
cannot state that the use of PV coupling minimizes the contact Lagrangians: while it is true for the isospin-even $\bar{\psi} \psi \Phi^{2}$ vertex, the isospin-odd contact Weinberg-Tomozawa interaction is actually close to zero in the representation with PS coupling. If one tries to include the heavy mesons the situation becomes even less clear: to include them one has to extend the symmetry in a model dependent way, e.g. to require the local $\mathrm{SU}(2) \times \mathrm{SU}(2)$ symmetry dynamically realized (and broken) either in the Young-Mills fashion or using the concept of hidden symmetry realization. The construction of these (approximately) chiral symmetric Lagrangians including heavy mesons is thus model dependent. It does not allow to formulate the consistent chiral counting scheme and even at the tree level the dynamical content has not been sufficiently constrained by detailed analysis of wide range of hadronic processes, which they in principle should describe.

In this paper we therefore consider only the Born amplitudes and the amplitudes with intermediate excitation of the Roper $N^{*}(1440)$ resonance, which have been proposed in Ref. 14], and have never been included in realistic calculations of trinucleon bound states. Since we conclude from the reasoning above that there is no strong reason to prefer the Born contributions obtained with PV $\pi N N$ coupling, we calculate also their PS version. In contrast with Ref. [14] we include all these potentials in $3 N$ Faddeev calculations exactly, i.e., without using perturbation theory. Their contributions to the binding energy are given individually.

Finally, we relate some of these short-range $3 N$ potentials to the corresponding counterterms from the chiral Lagrangian. We have deduced from the potentials with heavy meson exchange the effective contact low-energy four-nucleon-pion coupling constants and attempted to relate them with the constants of NLO interactions of ChPT [10, 15]. When the heavy meson propagator is reduced to a point, it appears that the pion-heavy-meson $3 N$ potentials with PS $\pi N N$ coupling are closer to those obtained from the contact lowenergy four-nucleon-pion NLO interactions of ChPT [10, 15] than their PV $\pi N N$ versions. Nevertheless, the comparison with chiral low energy constants is not straightforward, since some of the effective interactions we obtained by taking the point limit are not included in the NLO interactions of ChPT, and can be transformed to that form only after certain approximation. This approximation however does not seem to be numerically supported by results of our model calculations.

This paper is organized into four sections. Section II contains an overview on $3 N$ forces. 
Section III presents the long-long and the long-short $3 N$ forces and the numerical results. Section IV determines the low-energy constants and section V gives a summary and conclusions.

\section{BRIEF OVERVIEW OF $3 N$ FORCES}

Chiral symmetry has been recognized as an important guideline for the construction of nuclear forces, into which the $\pi N$ amplitude enters as one of its building blocks, long before the advent of ChPT. The process of incorporating constraints from chiral symmetry (breaking) into theoretical studies of the two-nucleon interaction was pioneered by G. Brown 16] in the early 1970's. Later, the same ideas were applied to the $3 N$ force, for which two different approaches were developed in parallel:

(i) One is based on the concept of partially conserved axial-vector current (PCAC) and the current algebra $(\mathrm{CA})$ formalism, built from equal-time commutation relations for vector and axial-vector currents. It underlies the well-known Tucson-Melbourne (TM) two-pionexchange $3 N$ force [11, 12] represented in Fig. 11. It goes beyond the static P-wave $\Delta$ contribution to the $\pi N$ amplitude, the only building block considered in its predecessor, the Fujita-Myazawa $3 N$ force. Current algebra and PCAC provide an elastic pion-nucleon scattering amplitude which includes the pion-nucleon $\sigma$-term, a direct measure of chiral symmetry breaking, which can be extracted from experiment.

(ii) The other approach stems from the so-called "effective chiral Lagrangians" for the $\pi N$ system. The first example of this approach is the Gell-Mann and Levy linear sigma model. Another example is the chiral Lagrangian with pseudo-vector $\pi N$ coupling, which underlies the construction of the so-called Brazilian 3N force in Ref. 17]. More recently, ChPT, based

on the Weinberg Lagrangian [18] supplemented by multinucleon contact terms, became a systematic way to approach two and $3 N$ forces [19], and was applied to the description of low-energy hadronic physics.

In particular, it was realized in Ref. [15] that although the construction of the TM twopion exchange $3 N$ force employs chiral constraints for $\pi N$ scattering through CA, it missed further constraints, which arise from embedding that amplitude in the $3 N$ system. The conclusion was that the TM form of the two-pion-exchange force contained a spurious term corresponding to contact terms between two nucleons and pions $(N N \pi \pi)$. This spurious 
term can be generated also in ChPT with the help of a pion field redefinition. But at the same time an additional two-nucleon contact term arises $(N N N N \pi)$, which has not been taken into account by the TM group and which exactly cancels the first spurious contact $(N N \pi \pi)$ contribution. As a net result, the so-called $c$ term of the TM force should be dropped and the so-called $a$ term is modified. We present here the effects of the chirally imposed changes to the TM force on the triton binding energy. A previous calculation of these effects [20] used a less realistic $N N$ interaction and a variational numerical method.

The CA program was extended to describe also $\pi N-\rho N$ transitions, from which a $\pi-\rho$ exchange $3 N$ force can be constructed. It seems natural to include such a mechanism, given the important interplay between $\pi$ and $\rho$ exchange in two-nucleon potentials. To model the $\pi-\rho 3 N$ force, one can use vector-meson dominance to access the $\rho$-analogue of the offmass-shell pion electroproduction. Chiral symmetry is in this case supplemented by gauge invariance to constrain the Ward identity amplitude.

The interest in the short-range $3 N$ forces increased in mid 1990's, when it was found that the lack of binding energy of the triton is not the only experimental signature of a $3 N$ force. According to 21] a spin-orbit structure of the $3 N$ force, not present in the standard two-pion exchange based forces, may solve systematic deviations between the theoretical and the experimental maximum of the nucleon vector analyzing power $A_{y}(\theta)$ in elastic $n d$ scattering below $30 \mathrm{MeV}$. The TM or the Brazilian $3 N$ force either produces no sizable effect or even worsens the disagreement with the data. But the study of Hüber et al. [9] indicated that the short-long range forces with a structure similar to that given by $\pi-\sigma$ and $\pi-\omega$ exchanges with an intermediate Roper resonance $N^{*}$ 14] and suggested by the NLO ChPT 15] could potentially improve the description of $A_{y}$. Similarly, a purely phenomenological spin-orbit $3 N$ force yields a noticeable improvement of the description of this observable 22].

More recently, Meissner et al. [23] actually claimed that the $A_{y}$ puzzle can be resolved by a chiral $N N$ force alone. However, the $N N$ interaction employed does not match the high quality of contemporary descriptions of $N N$ scattering data, in particular, for the crucial triplet P-waves [7, 21]. The NLO chiral potential gives a qualitative description of the phase shifts only for very small energies $(\leq 10 \mathrm{MeV})$, but for a truly quantitative fit one has to include NNLO corrections [6, 7]. Entem and Machleidt showed that no low- $\chi^{2} N N$ potential, neither one based on heavy-meson exchanges nor one constructed from ChPT, 
can solve the $A_{y}$ puzzle, since a good fit to the $N N$ phase shifts (in particular in the ${ }^{3} P_{J}$ channel) does not allow the variation needed to fix this spin observable. In a recent paper of Epelbaum et al. 10] it was found that NNLO $N N$ and leading order $3 N$ chiral potentials still do not solve the $A_{y}$ problem completely. Also the Urbana $3 N$ force (a Fujita-Myazawa force with a short-range regularization determined phenomenologically through a fit to the triton binding energy) does not provide a solution. An interesting alternative was suggested by Canton et al. [24], who constructed an additional $3 N$ force by reducing $\pi-3 N$ equations below the pion threshold. Their force is very similar in spin-isopsin structure to the $\pi-\sigma$ and $\pi-\omega$ force with intermediate Roper resonance discussed above, but it is multiplied by a part of an energy-dependent $N N$ T-matrix.

In addition to the mentioned $n d$ scattering problem, a good description of neutron-rich light nuclei is only possible [25] when a phenomenological $3 N$ interaction with three pion exchanges is added to the original Urbana force [26]. Finally, let us mention that while realistic $N N$ potentials alone predict an equilibrium density for symmetric nuclear matter that is too high, phenomenological $3 N$ forces can change it in the right direction [27].

This overall picture indicates that the long-range $\pi-\pi 3 N$ force needs to be complemented by other physical mechanisms.

\section{LONG-LONG AND LONG-SHORT RANGE $3 N$ FORCES}

The phenomenology of the $N N$ interaction shows that the most important meson exchanges in every realistic OBEP are exchanges of the pion, of the vector $\rho$ and $\omega$ mesons, and of the (fictitious) scalar $\sigma$ meson. Therefore, one should also investigate the role of such exchanges in the modeling of a $3 N$ force.

\section{A. $\pi-\pi$ exchange force}

Given the importance of the one-pion-exchange potential (OPEP) in determining the long-range part of the $N N$ interaction, it was natural that the first $3 N$ force considered was the two-pion exchange. The corresponding diagram is shown in Fig. 1k, where the blob represents all possible pion re-scattering processes. The iteration of the OPEP has to be subtracted from this Feynman diagram, since it is generated automatically by iterating 
the $N N$ potential in the Faddeev equation. The $\pi N$ amplitude $t_{\pi N}$ entering the $3 N$ force involves virtual pions. This off-mass-shell continuation is constrained by the soft pion lowenergy theorems. In this paper, we adopt the (modified) TM version of $\pi-\pi$ force, which has been constructed to explicitly conform with these requirements.

The TM $\pi-\pi 3 N$ force [11, 12] was generated by applying the Ward identities of CA to the amplitude of axial-vector current scattering on the nucleon. Using PCAC, the resulting $\pi N$ amplitude, in the even and odd isospin channels, was written as an expansion in powers of the momentum transfer $t$ and the crossing-variable $\nu=(s-u) / 4 M$. Then the "reality test" was applied with the successful result that CA predicts the first four coefficients of the expansion in good agreement with the empirical subthreshold expansion coefficients obtained from dispersion relations. Therefore, it was possible to use accurate empirical $\pi N$ data to construct the nearly model-independent TM force. The dispersion analysis has been updated over time with the inclusion of new data, therefore the coefficients of the TM force have also evolved.

In terms of physical mechanisms, the non-Born part of the TM $\pi N$ amplitude contains terms that can be interpreted as $t$-channel $\sigma$ and $\rho$ exchanges, as well as contributions from the $\Delta$ resonance in the intermediate state. The contributions of the subtracted nucleon Born diagrams were analyzed in detail in 28, 29] and it was later shown numerically that they are relatively small [30], at least as far as their contribution to the triton binding is concerned.

As argued in [15], the TM $\pi N$ amplitude, although constructed from CA and obeying the chiral constraints in the $\pi N$ sector, should not be simply attached to two additional nucleon lines, since that would be inconsistent with the chiral counting for the $3 N$ potential. More precisely, the TM $\pi N$ amplitude can be derived from the usual chiral Lagrangian after the pion field re-definition

$$
\boldsymbol{\pi}^{\prime}=\boldsymbol{\pi}(1-c) N^{\dagger} N
$$

where $c \approx \sigma /\left(m_{\pi}^{2} f_{\pi}^{2}\right)$ is the constant of the so-called TM $c$-term. The original chiral Lagrangian in the "natural" representation acquires after such redefinition two additional terms (relevant up to the order considered)

$$
\Delta \mathcal{L}^{(1)}=-c N^{\dagger} N\left\{\left(\boldsymbol{\pi}^{\prime} \partial_{\mu} \partial^{\mu} \boldsymbol{\pi}^{\prime}+m_{\pi}^{2} \boldsymbol{\pi}^{\mathbf{2}}\right)+\frac{g_{A}}{2 f_{\pi}}\left[\left(\boldsymbol{\nabla} N^{\dagger}\right) \cdot \boldsymbol{\sigma} \boldsymbol{\tau} \cdot \boldsymbol{\pi}^{\prime} N+N^{\dagger} \boldsymbol{\tau} \cdot \boldsymbol{\pi}^{\prime} \boldsymbol{\sigma} \cdot(\boldsymbol{\nabla} N)\right]\right\} .
$$

The TM amplitude includes the term corresponding to the first part of $\Delta \mathcal{L}^{(1)}$ (the $c$-term proportional to $\mathbf{q}_{2}^{2}+\mathbf{q}_{3}^{2}$ ), but in the construction of the TM $3 N$ potential the second term 
(which involves two nucleons) was not considered. Therefore, according to Ref. [15], the TM $c$-term should be dropped and its $a$-term should be replaced by $a^{\prime}=a-2 m_{\pi}^{2} c$. The TM $\pi-\pi$ force modified in this way was recently adopted in [20] and labeled TM'(99). However, we would like to point out that it is not correct to transform away the whole $c$ term, because it includes the contribution from the Born diagrams- that contribution arises naturally also in ChPT in its usual representation. Nevertheless, since this residual $c$-term is rather small and the $\pi-\pi$ force is not the main subject of interest of this paper, we avoid introducing further redefinitions of the TM force and we adopt TM'(99) for our numerical calculations.

Thus, the $\pi-\pi$ potential used in this paper reads

$$
\begin{aligned}
W_{\pi \pi}(1)= & -(2 \pi)^{3} \frac{g^{2}}{4 m^{3}}\left(\boldsymbol{\sigma}_{2} \cdot \mathbf{q}_{2}\right)\left(\boldsymbol{\sigma}_{3} \cdot \mathbf{q}_{3}\right) \tilde{\Delta}_{\pi}\left(\mathbf{q}_{2}\right) \tilde{\Delta}_{\pi}\left(\mathbf{q}_{3}\right) \\
& \times\left\{\left(\boldsymbol{\tau}_{2} \cdot \boldsymbol{\tau}_{3}\right)\left[a^{\prime}-b \mathbf{q}_{2} \cdot \mathbf{q}_{3}\right]-\left(i \boldsymbol{\tau}_{1} \cdot \boldsymbol{\tau}_{2} \times \boldsymbol{\tau}_{3}\right) d\left(i \boldsymbol{\sigma}_{1} \cdot \mathbf{q}_{2} \times \mathbf{q}_{3}\right)\right\}+2 \leftrightarrow 3,
\end{aligned}
$$

where we use for the meson momenta $\mathbf{q}_{i}=\mathbf{p}_{i}^{\prime}-\mathbf{p}_{i}$, so that they are always pointing away from the "active" nucleon as shown in Fig. 1p (to avoid confusion, we note that the different convention with the first pion incoming and the second outgoing from the active line rescattering "blob" is also often used in discussion of this potential). The overall momentum conservation is then given by $\mathbf{q}_{1}+\mathbf{q}_{2}+\mathbf{q}_{3}=0$. The functions $\tilde{\Delta}_{B}(\mathbf{q})$ are defined to contain the propagator function of the meson $B$ and the square of the strong $B N N$ form factor $\tilde{\Delta}_{B}(\mathbf{q})=F_{B N N}^{2}\left(\mathbf{q}^{2}\right) /\left(m_{B}^{2}+\mathbf{q}^{2}\right)$. The full potential contains additional four terms following from (3) by cyclic permutations.

The parameters of the TM'(99) force are given by

$$
\begin{aligned}
a^{\prime} & =\frac{\sigma}{f_{\pi}^{2}}-2 m_{\pi}^{2}\left(\frac{\sigma}{m_{\pi^{+}}^{2} f_{\pi}^{2}}-\frac{g^{2}}{4 m^{3}}+F_{\pi N N}^{\prime}(0) \frac{\sigma}{f_{\pi}^{2}}\right) \\
b & =-\frac{2}{m_{\pi^{+}}^{2}}\left[\bar{F}^{+}\left(0, m_{\pi^{+}}^{2}\right)-\frac{\sigma}{f_{\pi}^{2}}\right] \\
d & =-\left[\frac{\bar{B}^{-}(0,0)}{2 m}+\frac{g^{2}}{4 m^{3}}\right] .
\end{aligned}
$$

Here, $\sigma$ is the pion-nucleon sigma term, $g$ is the $\pi N N$ coupling constant, $f_{\pi}$ the pion decay constant, $\bar{F}^{+}(\nu, t)$ and $\bar{B}^{-}(\nu, t)$ are isospin-even non-spin flip and isospin-odd spin-flip $t$-channel $\pi N$ amplitudes, respectively, with the nucleon pole term subtracted [11]. Note that we distinguish between the mass of the charged pions, $m_{\pi^{+}}=139.6 \mathrm{MeV}$, and the isospin averaged pion mass $m_{\pi}=138.0 \mathrm{MeV}$. Although their difference is small, it affects 


\begin{tabular}{cr}
\hline \hline$\Lambda_{\pi N N} / m_{\pi}$ & $m_{\pi} a^{\prime}$ \\
\hline 4.1 & -1.203 \\
5.0 & -1.154 \\
5.8 & -1.127 \\
6.5 & -1.112 \\
7.1 & -1.101 \\
\hline \hline
\end{tabular}

TABLE I: Expansion coefficient $a^{\prime}$ of the $\pi \mathrm{N}$ amplitude used in the TM $\pi-\pi$ force as a function of the $\pi N N$ vertex cut-off parameter $\Lambda_{\pi N N}$, in units of the isospin averaged pion mass. The other coefficients $b$ and $d$ do not depend on $\Lambda_{\pi N N}$ and have the values $m_{\pi}^{3} b=-2.801$ and $m_{\pi}^{3} d=-0.754$.

the $3 \mathrm{~N}$ force parameters in a noticeable way. The vertex form factor

$$
F_{\pi N N}\left(\mathbf{q}^{2}\right)=\frac{\Lambda_{\pi N N}^{2}-m_{\pi}^{2}}{\Lambda_{\pi N N}^{2}+\mathbf{q}^{2}}
$$

depends on the cut-off parameter $\Lambda_{\pi N N}$, which in the original TM force was taken to be 5.8 $m_{\pi}$ in order to be consistent with a Goldberger-Treiman discrepancy of $3 \%$ determined at the time. Based on more recent data, the Goldberger-Treiman discrepancy shrank to about $2 \%$, which corresponds to $\Lambda_{\pi N N}$ close to $7.1 m_{\pi}$. On the other hand, $\Lambda_{\pi N N}$ is frequently tuned to reproduce the triton binding energy in calculations that include only the $\pi-\pi$ exchange part of the $3 N$ force. Such calculations require much lower values, close to $4 m_{\pi}$. Clearly, the functional form of the form factor is not much constrained by the knowledge of the coupling constant $g F_{\pi N N}\left(q^{2}\right)$ at the two points $q^{2}=0$ and $q^{2}=m_{\pi}^{2}$ and thus does not allow a strict determination of a cut-off mass. We keep therefore the by now traditional reference value $\Lambda_{\pi N N}=5.8 m_{\pi}$ as our standard one, but also vary it then between the limits indicated above to study the sensitivity of the results. In particular, we investigate the question whether the short-range forces are able to reduce the strong cut-off dependence, as one may expect from arguments of ChPT.

Adopting the values [20] $g^{2}=172.1, \bar{F}^{+}\left(0, m_{\pi^{+}}^{2}\right)=-0.05 m_{\pi^{+}}^{-1}, \bar{B}^{-}(0,0)=8.6 m_{\pi^{+}}^{-1}$,

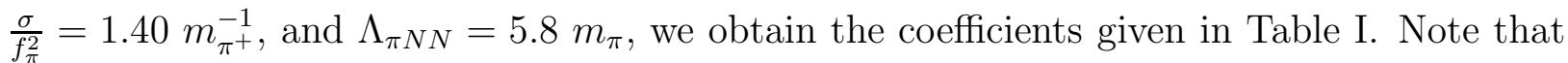
$a^{\prime}$ depends weakly on $\Lambda_{\pi N N}$, a dependence often ignored in practical calculations that vary the cut-off mass. 
While the rather strong dependence of the $3 N$ binding energy on $\Lambda_{\pi N N}$ has been recognized as a source of significant uncertainty, little attention has been paid to uncertainties originating from the experimental errors in the other parameters of the TM $\pi-\pi$ exchange force. Since the first publication of the TM force, the experimentally determined values of $g^{2}, \bar{F}^{+}\left(0, m_{\pi^{+}}^{2}\right), \bar{B}^{-}(0,0)$, and $\frac{\sigma}{f_{\pi}^{2}}$ have changed several times, leading each time to updated force parameters $a^{\prime}, b$, and $d$, according to Eqs. (4) to (6). We estimate here how these experimental uncertainties propagate into uncertainties of the triton binding energy $E_{t}$.

For simplicity, we introduce the dimensionless variables

$$
\begin{gathered}
x=\frac{\sigma}{f_{\pi}^{2}} m_{\pi^{+}}, \quad y=g^{2}, \quad z=\bar{F}^{+}\left(0, m_{\pi^{+}}^{2}\right) m_{\pi^{+}}, \quad u=\bar{B}^{-}(0,0) m_{\pi^{+}}^{2}, \\
\bar{\Lambda}=\frac{\Lambda}{\mu}, \quad r=\frac{\mu}{m_{\pi^{+}}}, \quad q=\frac{\mu}{m},
\end{gathered}
$$

as well as the dimensionless force parameters

$$
\bar{a}=a^{\prime} \mu, \quad \bar{b}=b \mu^{3}, \quad \bar{d}=d \mu^{3} .
$$

Using this notation, Eqs. (4) through (6) become

$$
\begin{aligned}
& \bar{a}=x r\left(1-2 r^{2}-2 \frac{\bar{\Lambda}^{2}-1}{\bar{\Lambda}^{4}}\right)+\frac{1}{2} q^{3} y, \\
& \bar{b}=-2 r^{3}(x-z), \\
& \bar{d}=-\frac{1}{4} q^{3} y-\frac{1}{2} r^{2} q u .
\end{aligned}
$$

Adding (independent) errors in quadrature, and taking into account the relations (11) through (13), we arrive at the following expression for the square of the uncertainty in $E_{t}$ due to the uncertainties $\Delta x, \Delta y, \Delta z$, and $\Delta u$ in $x, y, z$, and $u$ :

$$
\begin{aligned}
& \left(\Delta E_{t}\right)^{2}=\left[r\left(1-2 r^{2}-2 \frac{\bar{\Lambda}^{2}-1}{\bar{\Lambda}^{4}}\right) \frac{\partial E_{t}}{\partial \bar{a}}-2 r^{3} \frac{\partial E_{t}}{\partial \bar{b}}\right]^{2}(\Delta x)^{2}+ \\
& {\left[\frac{1}{2} q^{3}\left(\frac{\partial E_{t}}{\partial \bar{a}}-\frac{1}{2} \frac{\partial E_{t}}{\partial \bar{d}}\right)\right]^{2}(\Delta y)^{2}+\left[2 r^{3} \frac{\partial E_{t}}{\partial \bar{b}}\right]^{2}(\Delta z)^{2}+\left[\frac{1}{2} r^{2} q \frac{\partial E_{t}}{\partial \bar{d}}\right]^{2}(\Delta u)^{2} .}
\end{aligned}
$$

Note that it is simpler to vary the three constants $\bar{a}, \bar{b}$, and $\bar{d}$ instead of the four experimental values $x, y, z$, and $u$, but one has to keep in mind that the former are not independent from each other. 


\begin{tabular}{lcccc}
\hline \hline & Reid & Paris & Nijmegen 93 & Bonn B \\
\hline$\partial E_{t} / \partial \bar{a}$ & 0.080 & 0.090 & 0.095 & 0.095 \\
$\partial E_{t} / \partial \bar{b}$ & 0.725 & 0.745 & 0.845 & 0.750 \\
$\partial E_{t} / \partial \bar{d}$ & 0.545 & 0.470 & 0.540 & 0.505 \\
$\Delta E_{t}$ & 0.377 & 0.389 & 0.440 & 0.393 \\
$\Delta E_{t}^{\text {app }}$ & 0.362 & 0.372 & 0.423 & 0.375 \\
\hline \hline
\end{tabular}

TABLE II: Numerical results for the partial derivatives of the triton binding energy with respect to the TM $\pi-\pi$ force parameters and resulting uncertainties according to Eqs. (14) and (15).

The partial derivatives $\partial E_{t} / \partial \bar{a}, \partial E_{t} / \partial \bar{b}$, and $\partial E_{t} / \partial \bar{d}$, are calculated numerically for the standard set of parameters of Table \at $\bar{\Lambda}=5.8$. As estimates of the experimental uncertainties in $x$ and $z$ we use the values given in Ref. [20], $\Delta x=0.25$ and $\Delta z=0.05$. As a reasonable estimate for $\Delta y$ we choose the difference between the current value $y=172.1$ and the one used in the original TM force, $y=179.7$, yielding $\Delta y=7.6$. The value $u=8.1$ is given in [20] without indication of the error. Assuming that the specified digits are indeed significant, we set $\Delta u=0.1$.

The numerical results for the partial derivatives of $E_{t}$ with respect to $\bar{a}, \bar{b}$, and $\bar{d}$, and the corresponding values of $\Delta E_{t}$ are shown in Table $\amalg$ for the TM $\pi-\pi$ force in combination with various $N N$ potentials. In each case, $\Delta E_{t}$ is roughly $0.4 \mathrm{MeV}$. This is a significant value which clearly shows that the dependence on $\Lambda_{\pi N N}$ is not the only source of uncertainty in predictions of $E_{t}$.

A closer inspection of (14) reveals that $\Delta E_{t}$ is almost completely dominated by $\Delta x$, the uncertainty in the pion-nucleon sigma term. One can easily derive the approximate form

$$
\Delta E_{t}^{\mathrm{app}} \approx 2 \frac{\partial E_{t}}{\partial \bar{b}} \Delta x
$$

the results of which are also shown in Table 11 and come very close to the full result, confirming that the uncertainties in the other parameters are secondary as long as the pionnucleon sigma term is not determined with significantly better accuracy.

Since (15) is a lower limit of (14) one also has to conclude that currently predictions of the triton binding energy employing the TM force cannot be made with a better accuracy 
than plus or minus $0.4 \mathrm{MeV}$. Clearly, adding other contributions to the $3 N$ force will further increase this uncertainty.

\section{B. $\pi-\rho$ exchange force}

In the $N N$ potential, $\rho$-meson exchange provides important contributions to the tensor and spin-orbit components. Its role is enhanced by the large anomalous $\rho N N$ coupling $\kappa_{\rho}$, connected (via VMD) to the anomalous isovector magnetic moment of the nucleon. The $\pi-\rho$ exchange was therefore always considered the next most important part of the $3 N$ force [13, 29, 30] after $\pi-\pi$ exchange. In this paper we will take another look at its contributions stemming from the nucleon Born diagrams. The TM $\pi-\rho$ exchange force also includes processes with intermediate $\Delta$ resonance excitations, which numerically turn out to be equally important. In our calculations the $\pi-\rho$ potentials with intermediate $\Delta$ resonance are included in the form (and with the corresponding parameters) specified in Ref. [30].

The nucleon Born contributions to the $\pi-\rho 3 N$ force are derived in Appendix A. They depend significantly on the form of the $\pi N N$ coupling. For the PV $\pi N N$ coupling one gets

$$
\begin{aligned}
W_{\pi \rho T}^{\mathrm{PV}+}(1)=- & (2 \pi)^{3} \frac{g^{2} g_{\rho}^{2}}{4 m^{3}}\left[\left(\boldsymbol{\tau}_{2} \cdot \boldsymbol{\tau}_{3}\right) \boldsymbol{\sigma}_{1} \cdot \mathbf{q}_{3}-\left(i \boldsymbol{\tau}_{1} \cdot \boldsymbol{\tau}_{2} \times \boldsymbol{\tau}_{3}\right) \boldsymbol{\sigma}_{1} \cdot \mathbf{Q}_{1}\right] \\
& \times \boldsymbol{\sigma}_{2} \cdot \mathbf{q}_{2} \tilde{\Delta}_{\pi}\left(\mathbf{q}_{2}\right) \tilde{\Delta}_{\rho}\left(\mathbf{q}_{3}\right)+2 \leftrightarrow 3, \\
W_{\pi \rho S}^{\mathrm{PV}+}(1)=0 &
\end{aligned}
$$

whereas for the PS $\pi N N$ coupling

$$
\begin{gathered}
W_{\pi \rho T}^{\mathrm{PS}-}(1)=-(2 \pi)^{3} \frac{g^{2} g_{\rho}^{2}\left(1+\kappa_{\rho}\right)}{4 m^{3}}\left(\boldsymbol{\tau}_{2} \cdot \boldsymbol{\tau}_{3}\right) \boldsymbol{\sigma}_{1} \cdot \mathbf{q}_{3} \boldsymbol{\sigma}_{2} \cdot \mathbf{q}_{2} \tilde{\Delta}_{\pi}\left(\mathbf{q}_{2}\right) \tilde{\Delta}_{\rho}\left(\mathbf{q}_{3}\right)+2 \leftrightarrow 3, \\
W_{\pi \rho S}^{\mathrm{PS}-}(1)=+(2 \pi)^{3} \frac{g^{2} g_{\rho}^{2}}{4 m^{3}}\left(i \boldsymbol{\tau}_{1} \cdot \boldsymbol{\tau}_{2} \times \boldsymbol{\tau}_{3}\right)\left[\boldsymbol{\sigma}_{1} \cdot \mathbf{Q}_{3}+\left(1+\kappa_{\rho}\right) i \boldsymbol{\sigma}_{1} \times \boldsymbol{\sigma}_{3} \cdot \mathbf{q}_{3}\right] \\
\quad \times \quad \boldsymbol{\sigma}_{2} \cdot \mathbf{q}_{2} \tilde{\Delta}_{\pi}\left(\mathbf{q}_{2}\right) \tilde{\Delta}_{\rho}\left(\mathbf{q}_{3}\right)+2 \leftrightarrow 3
\end{gathered}
$$

where again $\mathbf{q}_{i}=\mathbf{p}_{i}^{\prime}-\mathbf{p}_{i}, \mathbf{Q}_{i}=\mathbf{p}_{i}^{\prime}+\mathbf{p}_{i}$. The subscripts $S$ and $T$ refer to the exchange of the space and time components of the $\rho$ field, respectively. For brevity, we will call the corresponding terms "spacelike" and "timelike". The superscripts PS and PV correspond to the type of $\pi N N$ coupling, the superscripts "+" stand for the contribution of the positive energy nucleon Born diagrams as derived in Appendix A, superscripts "-" denote the "true" pair (or Z-diagram) terms. 


\begin{tabular}{llccc}
\hline \hline Meson & mass $(\mathrm{MeV})$ & $\frac{g^{2}}{4 \pi}$ & $\frac{g^{* 2}}{4 \pi}$ & cut-off \\
\hline$\pi$ & 138 & 13.6953 & 2.1664 & $\Lambda_{\pi N N}=\Lambda_{\pi N N^{*}}=5.8 m_{\pi}$ \\
$\rho$ & 768.3 & 0.81 & & $\Lambda_{\rho N N}($ Dirac $)=12 m_{\pi}$ \\
& & & $\Lambda_{\rho_{N} N}($ Pauli $)=7.4 m_{\pi}$ \\
$\sigma$ & 584 & 10.3251 & 0.6 & $\Lambda_{\sigma N N}=\Lambda_{\sigma N N^{*}}=1995 \mathrm{MeV}$ \\
$\omega$ & 782.6 & 24.5 & 1.4237 & $\Lambda_{\omega N N}=\Lambda_{\omega N N^{*}}=1850 \mathrm{MeV}$ \\
\hline \hline
\end{tabular}

TABLE III: Masses, couplings, and cut-off parameters that appear in the $3 N$ forces. For the $\rho$ and the $\omega$ mesons there are also the tensor/vector coupling ratios $\kappa_{\rho}=6.6$ and $\kappa_{\omega}=0.0$, respectively. The parameters of the $\pi$ and $\rho$ mesons are taken from the TM'(99) force and from Ref. [30], the parameters of the $\omega$ meson from the Bonn B potential. The $\sigma$ meson parameters are determined in Appendix B

In the numerical calculations we keep the $\pi-\rho$ force parameters of [13, 30], with the exception of $g^{2}$ which is updated to the value of TM'(99) (see Table III).

The terms proportional to $\mathbf{Q}_{i}$ are non-local and have been omitted so far from $3 N$ potentials, mainly because of the difficulties associated with performing calculations with non-local interactions in coordinate space. Working in momentum space, we are in principle not hampered by non-localities. However, in this paper we focus on the relation between traditional meson-theoretical $3 N$ forces and the $3 N$ forces derived from ChPT where non-local terms are discarded. Hence it would not be useful to keep those terms in our calculations, and we neglect them as well.

The above results illustrate one important point: One cannot rely on the nucleon Born terms alone to yield the most important contribution to the $3 N$ force. In particular, it would be incorrect to claim that since the PV $\pi N N$ coupling is "more consistent" with the important chiral symmetry requirement, one should use the above potentials $W_{\pi \rho T}^{\mathrm{PV}+}$ and $W_{\pi \rho S}^{\mathrm{PV}+}$. Indeed, the construction of the $\pi-\rho 3 N$ force by the TM group [13] via the extension of CA to include vector meson dominance, has shown that its most important part - the so called Kroll-Ruderman (KR) term - follows (in the theory with PV coupling) not from the nucleon Born term, but from an additional contact term required by gauge invariance of the pion photoproduction amplitude. This is, of course, very similar to the 
situation for the leading order isovector meson exchange currents: there the nucleon Born term with PS coupling gives the most important contribution (which is supplemented by the pion-in-flight diagram), whereas the Born term for PV coupling does not contribute and gauge invariance requires the presence of the contact interaction. The KR contact term happens to be opposite in sign to the chiral contact term, arising from the chiral rotation from the PV to PS coupling and introduced in Appendix A. Thus, it appears reasonable to adopt for the $\pi-\rho S$ exchange potential just that KR term, i.e., to take

$$
W_{\pi \rho S}^{K R}=-W_{\pi \rho S}^{\mathrm{cont}}=W_{\pi \rho S}^{\mathrm{PS}-}
$$

But then it would seem natural to take for the timelike exchange the corresponding $W_{\pi \rho T}=$ $W_{\pi \rho T}^{\mathrm{PS}-}$, which is enhanced by the large factor $1+\kappa_{\rho}$ compared to $W_{\pi \rho T}^{\mathrm{PV}+}$. Note, that in 14$]$ (in which the Born contributions to the $\pi-\rho S$ where first derived) an expression identical to $W_{\pi \rho T}^{\mathrm{PV}+}$ is listed for the timelike part, while $W_{\pi \rho S}^{\mathrm{PS}-}$ (the $\mathrm{KR}$ term) is given for the spacelike $\operatorname{part}^{1}$.

We do not intend to imply by the discussion above that the PS $\pi N N$ coupling is to be preferred in this context. In fact, trying to make any preference for the Lagrangian involving heavy mesons is meaningless (unless one attempts to extend the global chiral symmetry to a local one and builds a model which provides all relevant contact terms [31]). We only want to point out that more simplistic approaches (like the one of this paper and that of Ref. 14]: taking only the Born terms with a hope that they contain the most important effects) do not give an unambiguous prediction. Besides, the $\rho-T$ contribution can be comparable to others when it is enhanced by the large factor $1+\kappa_{\rho}$, as it happens for PS coupling.

\section{C. $\pi-\sigma$ and $\pi-\omega$ exchange force}

Since $\sigma$ and $\omega$ meson exchanges play an important role in OBE models of the $N N$ interaction, they contribute naturally also to the $3 N$ force. Short-range $\pi-\sigma$ and $\pi-\omega 3 N$ potentials were introduced in Ref. [14]. They are derived from diagrams with intermediate positive-energy nucleons with PV $\pi N N$ coupling.

\footnotetext{
1 There are, unfortunately, two misprints in Eq. (2.8) of Ref. [14]: a factor of $\left(1+\kappa_{\rho}\right)$ is missing in the second term and the first term has an extra factor 2 .
} 
Unlike in the $\pi-\pi$ force, the Born terms in $\pi-\sigma$ and $\pi-\omega$ potentials (Fig. (1a) are rather large. As the $\pi-\rho$ potentials from the previous section, they depend on the type of $\pi N N$ coupling. We also include additional $\sigma$ and $\omega$ exchange contributions generated by excitations of the intermediate nucleon to the Roper resonance [14] (Fig. 1b).

The $\pi-\sigma$ and $\pi-\omega$ potentials corresponding to the nucleon Born diagrams follow immediately from the expressions developed in Appendix [ . For $\pi-\sigma$ exchange we get

$$
\begin{aligned}
W_{\pi \sigma}^{\mathrm{PV}+}(1) & =+(2 \pi)^{3} \frac{g^{2} g_{\sigma}^{2}}{4 m^{3}}\left(\boldsymbol{\tau}_{1} \cdot \boldsymbol{\tau}_{2}\right) \boldsymbol{\sigma}_{1} \cdot \mathbf{q}_{3} \boldsymbol{\sigma}_{2} \cdot \mathbf{q}_{2} \tilde{\Delta}_{\pi}\left(\mathbf{q}_{2}\right) \tilde{\Delta}_{\sigma}\left(\mathbf{q}_{3}\right)+2 \leftrightarrow 3, \\
W_{\pi \sigma}^{\mathrm{PS}-}(1) & =-(2 \pi)^{3} \frac{g^{2} g_{\sigma}^{2}}{4 m^{3}}\left(\boldsymbol{\tau}_{1} \cdot \boldsymbol{\tau}_{2}\right) \boldsymbol{\sigma}_{1} \cdot \mathbf{q}_{2} \boldsymbol{\sigma}_{2} \cdot \mathbf{q}_{2} \tilde{\Delta}_{\pi}\left(\mathbf{q}_{2}\right) \tilde{\Delta}_{\sigma}\left(\mathbf{q}_{3}\right)+2 \leftrightarrow 3,
\end{aligned}
$$

and for $\pi-\omega$ exchange

$$
\begin{aligned}
W_{\pi \omega}^{\mathrm{PV}+}(1) & =-(2 \pi)^{3} \frac{g^{2} g_{\omega}^{2}}{4 m^{3}}\left(\boldsymbol{\tau}_{1} \cdot \boldsymbol{\tau}_{2}\right) \boldsymbol{\sigma}_{1} \cdot \mathbf{q}_{3} \boldsymbol{\sigma}_{2} \cdot \mathbf{q}_{2} \tilde{\Delta}_{\pi}\left(\mathbf{q}_{2}\right) \tilde{\Delta}_{\omega}\left(\mathbf{q}_{3}\right)+2 \leftrightarrow 3, \\
W_{\pi \omega}^{\mathrm{PS}-}(1) & =-(2 \pi)^{3} \frac{g^{2} g_{\omega}^{2}\left(1+\kappa_{\omega}\right)}{4 m^{3}}\left(\boldsymbol{\tau}_{1} \cdot \boldsymbol{\tau}_{2}\right) \boldsymbol{\sigma}_{1} \cdot \mathbf{q}_{3} \boldsymbol{\sigma}_{2} \cdot \mathbf{q}_{2} \tilde{\Delta}_{\pi}\left(\mathbf{q}_{2}\right) \tilde{\Delta}_{\omega}\left(\mathbf{q}_{3}\right)+2 \leftrightarrow 3 .
\end{aligned}
$$

In Ref. [14], only the potentials for PV coupling are given. Since $\kappa_{\omega}$ is very small, the results for $\pi-\omega$ do not depend much on the type of the $\pi N N$ coupling. However, the $\pi-\sigma$ potentials do differ. For PV coupling, the $\pi-\sigma$ and $\pi-\omega$ potentials $W_{\pi \sigma}^{\mathrm{PV}+}(1)$ and $W_{\pi \omega}^{\mathrm{PV}+}(1)$ have identical structure but opposite sign. Therefore, as in the case of the $N N$ interaction, a strong cancellation between the $\pi-\sigma$ and $\pi-\omega 3 N$ interactions occurs. For small momenta, the sum of these potentials is proportional to

$$
\frac{g_{\sigma}^{2}}{m_{\sigma}^{2}}-\frac{g_{\omega}^{2}}{m_{\omega}^{2}},
$$

just as for the corresponding $N N$ potentials. For the potentials with PS coupling, Eqs. (22 24), such a cancellation does not take place. However, they would cancel if the potential (24) did not change much when the momentum $\mathbf{q}_{3}$ is replaced by the $-\mathbf{q}_{2}$. As discussed later, this is exactly the momentum replacement needed to extract the low-energy constants (LECs) of ChPT. Therefore we calculate the $\pi-\sigma$ potential in both forms. The extent of the cancellations between $\sigma$ and $\omega$ exchanges depends also on the numerical values of the masses and coupling constants of the $\sigma$ and $\omega$ mesons.

While we are trying to parameterize the $3 N$ forces as consistent as possible with the $N N$ potentials they are combined with, we face a problem with the $\sigma$ meson. The only $N N$ potential with explicit $\sigma$ exchange we are using is Bonn B. Its $\sigma$ meson, however, is not a 
pure scalar-isoscalar particle, but has also a scalar-isovector component. This is reflected in the fact that the $\sigma$ mass, the $\sigma N N$ coupling constant and cut-off mass is different in $N N$ isospin 0 and 1 channels.

In order to get a true OBE representation of $\sigma$-exchange, we keep for the $3 N$ potential only the part of the Bonn $\sigma$ meson which corresponds to pure isoscalar exchange. The parameters of our $\sigma$ are then related to the Bonn $\sigma$ such that a simple OBE form, consistent with the way it is implemented in the $3 N$ forces, is obtained. Details of this procedure can be found in Appendix B.

The forces originated from excitations of the Roper resonance read

$$
\begin{aligned}
& W_{\pi \sigma}^{*}(1)=-(2 \pi)^{3} \frac{g g^{*} g_{\sigma} g_{\sigma}^{*}}{2\left(m^{*}-m\right) m^{2}}\left(\boldsymbol{\tau}_{1} \cdot \boldsymbol{\tau}_{2}\right) \boldsymbol{\sigma}_{1} \cdot \mathbf{q}_{2} \boldsymbol{\sigma}_{2} \cdot \mathbf{q}_{2} \tilde{\Delta}_{\pi}\left(\mathbf{q}_{2}\right) \tilde{\Delta}_{\sigma}\left(\mathbf{q}_{3}\right)+2 \leftrightarrow 3, \\
& W_{\pi \omega}^{*}(1)=+(2 \pi)^{3} \frac{g g^{*} g_{\omega} g_{\omega}^{*}}{2\left(m^{*}-m\right) m^{2}}\left(\boldsymbol{\tau}_{1} \cdot \boldsymbol{\tau}_{2}\right) \boldsymbol{\sigma}_{1} \cdot \mathbf{q}_{2} \boldsymbol{\sigma}_{2} \cdot \mathbf{q}_{2} \tilde{\Delta}_{\pi}\left(\mathbf{q}_{2}\right) \tilde{\Delta}_{\omega}\left(\mathbf{q}_{3}\right)+2 \leftrightarrow 3 .
\end{aligned}
$$

Here $m^{*}$ is the mass of the $N^{*}(1440)$ resonance. We would like to stress that the form of these potentials depends on the structure of the $N N^{*} \sigma$ and $N N^{*} \omega$ vertices. We adopted the very simple choice used in Ref. [14]. The form of these effective vertices follows from the quantum numbers of the baryon state $N^{*}$ and of the $\sigma$ and $\omega$ mesons, neglecting any possible dependence on the substructure of these particles. Even for the simple choice of the $N^{*}$ vertices of [14], theoretical predictions of the corresponding coupling constants (and their ratios) from quark models are hampered by the uncertain quark content of $N^{*}$, whereas their extraction from experimental data can be done only in an indirect and model-dependent way.

The strength of the $\pi N N^{*}$ coupling is calculated from the partial decay width of $N^{*} \rightarrow$ $N+\pi$. The recent determination [32] based on the value $\Gamma\left(N^{*} \rightarrow N \pi\right)=228 \mathrm{MeV}_{\text {gives }}^{2}$

$$
\frac{f_{\pi N N^{*}}^{2}}{4 \pi}=0.0117, \quad g^{*}=\frac{2 m}{m_{\pi}} f_{\pi N N^{*}}=5.22 .
$$

The couplings for the $\sigma N N^{*}$ and $\omega N N^{*}$ vertices are more difficult to pin down. The coupling constant of $\sigma N N^{*}$, extracted from the partial decay width of $N^{*} \rightarrow N+(\pi \pi)_{S-\text { wave }}^{I=0}$, depends critically on the assumed mass and width of the $\sigma$ resonance [32]. The " $\sigma$ meson" (with zero width) employed in the parameterization of the OBE potentials simulates not only $(\pi \pi)_{S-\text { wave }}^{I=0}$, but also other scalar-isoscalar exchanges. Such a phenomenological $\sigma$ exchange

\footnotetext{
${ }^{2}$ Equation (3.3.) of Ref. [14] is incorrect and the corresponding value of $\frac{f_{\pi N N^{*}}^{2}}{4 \pi}$ is overestimated by a factor of 3 .
} 
was used in a recent analysis [33] of the inelastic scattering $\alpha+p \rightarrow \alpha+X$, from where the effective coupling constant of $\sigma N N^{*}$ was extracted as

$$
\frac{g_{\sigma}^{* 2}}{4 \pi}=1.33
$$

with $m_{\sigma}=550$. This is much larger than typical values obtained from the Roper resonance decay [32].

On the other hand, Ref. [14] extracts $\frac{g_{\sigma}^{* 2}}{4 \pi}=0.1$, which is even smaller than all values of Ref. 32]. Given this wide spread of coupling constants in the literature, we adopt the intermediate $\frac{g_{\sigma}^{* 2}}{4 \pi}=0.6$ as our standard value, but calculate also the two extreme cases in order to observe the sensitivity of the results with respect to the choice of this parameter.

For $\omega N N^{*}$ we follow Ref. [14] and determine $g_{\omega}^{*}$ from the ratio

$$
g_{\omega}^{*}=g_{\omega} \frac{g_{\sigma}^{*}}{g_{\sigma}}
$$

which follows from a naive constituent quark model. The sum of the $3 N$ potential with intermediate Roper resonance and $\pi-\sigma$ and $\pi-\omega$ exchanges is then roughly proportional to

$$
-\frac{g_{\sigma}^{*}}{g_{\sigma}}\left(\frac{g_{\sigma}^{2}}{m_{\sigma}^{2}}-\frac{g_{\omega}^{2}}{m_{\omega}^{2}}\right) .
$$

Therefore we can expect the same amount of cancellation between $\sigma$ and $\omega$ terms in $3 N$ forces with Roper excitations as in $N N$ potential and $3 N$ Born contributions. However, since the simple scaling rule of coupling constants is theoretically not very well founded, we also use sets of parameters that do not satisfy (30).

\section{Numerical results}

We calculated the triton binding energies and $3 N$ wave functions for Hamiltonians containing both $N N$ and $3 N$ potentials by solving the non-relativistic $3 N$ Faddeev equations in momentum space exactly.

The $3 \mathrm{~N}$ potentials are expressed in terms of Jacobi momenta in the $\mathrm{cm}$ frame of the $3 \mathrm{~N}$ system, and decomposed into partial waves in a basis of $j j$ coupling states. Because of the complexity of the resulting numerical problem, we restrict ourselves to those partial waves in which the $N N$ pair total angular momentum does not exceed 2, which corresponds to 


\begin{tabular}{lcccccccc}
\hline \hline & \multicolumn{2}{c}{ Reid } & \multicolumn{2}{c}{ Paris } & \multicolumn{2}{c}{ Nijmegen 93} & \multicolumn{2}{c}{ Bonn B } \\
$3 \mathrm{NF}$ & $E_{t}$ & $\Delta E_{t}$ & $E_{t}$ & $\Delta E_{t}$ & $E_{t}$ & $\Delta E_{t}$ & $E_{t}$ & $\Delta E_{t}$ \\
\hline no $3 \mathrm{NF}$ & -7.230 & & -7.383 & & -7.756 & & -8.100 \\
$+\pi \pi\left(a^{\prime}\right)$ & -7.279 & -0.049 & -7.439 & -0.056 & -7.811 & -0.055 & -8.159 & -0.059 \\
$+\pi \pi(b)$ & -8.739 & -1.460 & -8.939 & -1.500 & -9.471 & -1.660 & -9.624 & -1.465 \\
$+\pi \pi(d)$ & -9.100 & -0.361 & -9.220 & -0.281 & -9.782 & -0.311 & -9.847 & -0.223 \\
$+\pi \rho(\mathrm{KR})$ & -9.017 & 0.083 & -9.118 & 0.102 & -9.635 & 0.147 & -9.672 & 0.175 \\
$+\pi \rho\left(\Delta^{+}\right)$ & -8.849 & 0.168 & -8.961 & 0.157 & -9.464 & 0.171 & -9.506 & 0.166 \\
$+\pi \rho\left(\Delta^{-}\right)$ & -8.747 & 0.102 & -8.821 & 0.140 & -9.285 & 0.179 & -9.325 & 0.181 \\
$+\pi \rho(T)$ & -8.772 & -0.025 & -8.850 & -0.029 & -9.316 & -0.031 & -9.352 & -0.027 \\
$+\pi \sigma(Z)$ & -8.273 & 0.499 & -8.213 & 0.637 & -8.663 & 0.653 & -8.658 & 0.694 \\
$+\pi \sigma\left(N^{*}\right)$ & -8.711 & -0.438 & -8.610 & -0.397 & -9.145 & -0.482 & -9.055 & -0.397 \\
$+\pi \omega(Z)$ & -9.213 & -0.502 & -9.380 & -0.770 & -9.977 & -0.832 & -9.956 & -0.901 \\
$+\pi \omega\left(N^{*}\right)$ & -8.735 & 0.478 & -8.898 & 0.482 & -9.370 & 0.607 & -9.524 & 0.432 \\
\hline \hline
\end{tabular}

TABLE IV: Triton binding energies and their differences (in $\mathrm{MeV}$ ) calculated for various model Hamiltonians with different $N N$ potentials and contributions to the $3 N$ force added consecutively. All $\pi N N$ vertices in the $3 N$ forces of this table are calculated in $\mathrm{PV}$ coupling. The columns labeled $E_{t}$ show the triton binding energies, while the ones labeled $\Delta E_{t}$ indicate the differences between the binding energies of consecutive rows, indicating the effect of the corresponding $3 N$ force component.

18 different three-body channels. Details of the formalism and the numerical methods are described in Ref. [34].

The main purpose of these calculations is to compare the effects of the various contributions to the $3 N$ force which were described in the previous sections on the triton binding energy. In order to study also the dependence of our results on the $N N$ interaction included in the Hamiltonian, we used a number of different $N N$ potentials, namely Reid [38], Paris [39], Nijmegen 93 [2], and Bonn B [40].

In Tables [V] and $\mathrm{V}$ we show the triton binding energies for various Hamiltonians where 
the $3 N$ forces of the previous sections are added successively. The binding energy differences depend somewhat on the order in which the potentials are added, therefore we later also show their expectation values.

\begin{tabular}{lcccccccc}
\hline \hline & \multicolumn{2}{c}{ Reid } & \multicolumn{2}{c}{ Paris } & \multicolumn{2}{c}{ Nijmegen 93} & \multicolumn{2}{c}{ Bonn B } \\
$3 \mathrm{NF}$ & $E_{t}$ & $\Delta E_{t}$ & $E_{t}$ & $\Delta E_{t}$ & $E_{t}$ & $\Delta E_{t}$ & $E_{t}$ & $\Delta E_{t}$ \\
\hline$+\pi \rho(T)$ & -8.859 & -0.112 & -8.953 & -0.132 & -9.425 & -0.140 & -9.452 & -0.127 \\
$+\pi \sigma(Z)$ & -10.492 & -1.633 & -10.544 & -1.591 & -11.431 & -2.006 & $-10.879^{a}$ & -1.427 \\
$+\pi \sigma\left(N^{*}\right)$ & -11.219 & -0.727 & -11.264 & -0.720 & $-12.215^{a}$ & -0.784 & $-11.600^{a}$ & -0.721 \\
$+\pi \omega(Z)$ & -13.680 & -2.461 & -15.367 & -4.103 & -20.952 & -8.737 & $-15.811^{a}$ & -4.211 \\
$+\pi \omega\left(N^{*}\right)$ & -12.674 & 1.006 & -14.134 & 1.233 & -16.869 & 4.083 & -18.345 & -2.534 \\
\hline \hline
\end{tabular}

${ }^{a}$ In addition to the specified result, another unphysical, deeply bound solution was obtained in this case.

TABLE V: Triton binding energies and their differences (in MeV), as in Table IV but with $\pi N N$ PS coupling in the $3 N$ forces. The binding energy differences in the first row are calculated with respect to the corresponding entries in the row labeled $+\pi \rho\left(\Delta^{-}\right)$of Table IV] Note that only for $\pi \rho(T)$ and $\pi \sigma(Z)$ the $3 N$ potentials using PS and PV coupling actually differ. The effects of the other, unchanged $3 N$ potentials on the binding energy are amplified compared to the case of PV coupling, since the $3 N$ wave functions are significantly altered by the $\pi \rho(T)$ and $\pi \sigma(Z)$ PS potentials.

First we make a few comments to the results with the $\pi-\pi 3 N$ potential only. In our calculations [30] with the old version of the TM force $\operatorname{TM}(93)$, which contains the $c$-term and uses somewhat different values of the constants $a, b$ and $d$, we obtained $E_{t}($ Reid $)=-8.904$ $\mathrm{MeV}$ and $E_{t}$ (Paris) $=-9.060 \mathrm{MeV}$. This means that transforming the $c$-term away and using the new version TM'(99), as in Table IV brings an extra binding of about $200 \mathrm{keV}$. The first line of Table VI shows that, even after removing the singular $c$-term, the TM $\pi-\pi$ force remains highly non-perturbative. Also the cut-off dependence of $E_{t}$ (see Fig. 2) remains about the same (see Refs. [20, 30]) as with the old version TM(93).

Most of the new short-range potentials considered in this paper contribute individually as much as (or more than) the TM $\pi-\rho$ short-range forces considered before. The only exception is the $\rho-T$ term, also not considered before. It is the only attractive part of the 


\begin{tabular}{lrrrrc}
\hline \hline $3 N$ potential & \multicolumn{1}{c}{$\Delta E_{t}$} & no $3 N$ force & with $\pi \pi$ & with $\pi \pi+\pi \rho$ & with $\pi \pi+\pi \rho+\pi \sigma+\pi \omega$ \\
\hline$\pi \pi$ & -1.837 & -1.281 & -2.572 & -2.190 & -2.274 \\
$\pi \rho$ & 0.370 & 0.139 & 0.450 & 0.293 & 0.317 \\
$\pi \sigma$ & 0.240 & 0.275 & 0.748 & 0.605 & 0.881 \\
$\pi \omega$ & -0.288 & -0.228 & -0.758 & -0.594 & -0.968 \\
\hline \hline
\end{tabular}

TABLE VI: Matrix elements of the $3 N$ potentials of the first column calculated with different wave functions. For comparison, the column labeled $\Delta E_{t}$ shows the binding energy differences according to Table IV] All wave functions are calculated with the Paris potential and various $3 N$ forces in the Hamiltonian.

$\pi-\rho 3 N$ force, but it is about 5 times smaller than the KR term. Only in PS coupling the $\rho-T$ term is enhanced by the factor $1+\kappa_{\rho}$ and becomes comparable to the KR.

The $\sigma$ and $\omega$ exchange contributions are rather large. The Born terms are repulsive in case of the $\sigma$ and attractive for the $\omega$, but when PV $\pi N N$ coupling is used they cancel in a similar way as the corresponding $N N$ potentials. For the PS coupling, the $\pi-\sigma$ Born contribution turns attractive and together the $\pi-\sigma$ and $\pi-\omega$ exchanges lead to strong overbinding, as Table $\nabla$ demonstrates. Nevertheless, it would be premature to rule out the PS coupling solely based on these results: we would like to remind that other non-Born contact terms have not been investigated so far and are likely to be as important as the Born term considered here.

One example of such contributions are forces with an intermediate Roper resonance. We observe that individually they are also rather large. However, as we have already pointed out above, there are significant uncertainties in the coupling constants involving $N^{*}$, and the effect of those potentials can vary accordingly. Nevertheless, it is remarkable that even for our smallest value of $g_{\sigma}^{*}$ the $\pi-\sigma N^{*}$ term is comparable to the $\pi-\rho \Delta$ forces, as can be seen by comparing Tables IV and VII Although the effect of the $\pi-\sigma N^{*}$ force scales with $g_{\sigma}^{*}$, the overall contribution of $\pi-\sigma N^{*}$ and $\pi-\omega N^{*}$ forces does not change much, since we assume the constituent quark model ratio $g_{\sigma}^{*} / g_{\omega}^{*}$, which leads to a strong cancellation between them. Removing this constraint, as for instance in the last line of Table VII can have an effect on the overall contribution of $\pi-\sigma$ and $\pi-\omega$ forces comparable to the 


\begin{tabular}{|c|c|c|c|c|c|c|}
\hline \multirow[b]{2}{*}{$3 \mathrm{NF}$} & \multicolumn{2}{|c|}{$\frac{g_{\sigma}^{*}}{4 \pi}=0.1$} & \multicolumn{2}{|c|}{$\frac{g_{\sigma}^{*}}{4 \pi}=0.6$} & \multicolumn{2}{|c|}{$\frac{g_{\sigma}^{*}}{4 \pi}=1.33$} \\
\hline & $E_{t}$ & $\Delta E_{t}$ & $E_{t}$ & $\Delta E_{t}$ & $E_{t}$ & $\Delta E_{t}$ \\
\hline$\pi \pi+\pi \rho$ & -8.850 & & -8.850 & & -8.850 & \\
\hline$+\pi \sigma(Z)$ & -8.213 & 0.637 & -8.213 & 0.637 & -8.213 & 0.637 \\
\hline$+\pi \sigma\left(N^{*}\right)$ & -8.369 & -0.156 & -8.610 & -0.397 & -8.822 & -0.609 \\
\hline$+\pi \omega(Z)$ & -9.073 & -0.704 & -9.380 & -0.770 & -9.652 & -0.830 \\
\hline \multicolumn{7}{|c|}{ with $g_{\omega}^{*}=g_{\omega} \frac{g_{\sigma}^{*}}{g_{\sigma}}$} \\
\hline$+\pi \omega\left(N^{*}\right)$ & -8.883 & 0.190 & -8.898 & 0.482 & -8.910 & 0.742 \\
\hline \multicolumn{7}{|c|}{ with $\frac{g_{\omega}^{*}}{4 \pi}=0.24$} \\
\hline$+\pi \omega\left(N^{*}\right)$ & -8.882 & 0.191 & -9.174 & 0.206 & -9.431 & 0.221 \\
\hline
\end{tabular}

TABLE VII: Triton binding energies and their differences (in $\mathrm{MeV}$ ), as in Table IV for the Paris $N N$ potential and $3 N$ potentials with different coupling constants $g_{\sigma}^{*}$ and $g_{\omega}^{*}$. The first two lines are taken from Table IV to define reference values. The contribution of $\pi \sigma\left(N^{*}\right)$ increases with increasing $g_{\sigma}^{*}$, and so does the contribution of $\pi \omega\left(N^{*}\right)$ in the fifth line for a fixed ratio $g_{\omega}^{*} / g_{\sigma}^{*}$, keeping the binding energy almost constant. When $g_{\omega}^{*}$ is kept fixed (last line), these cancellations no longer take place and the binding energy increases.

transition from PV to PS coupling in Z-digrams: the cancellation between $\pi-\sigma N^{*}$ and $\pi-\omega N^{*}$ forces could become much weaker or disappear and very pronounced changes of $E_{t}$ can be expected. Having more reliable information on $g_{\omega}^{*} / g_{\sigma}^{*}$ is thus absolutely crucial for more definite conclusion on the importance of the short-range $3 N$ forces considered in this paper.

In Table VIII we show the expectation values calculated with the wavefunction obtained from the Hamiltonian with all $3 N$ potentials in PV coupling. The expectation values of the $\pi-\pi$ potential and of the full $3 N$ potential do not vary much, but the individual forces show a rather strong dependence on the $N N$ potential used. Our results (Tables VI and VIII) for the total $\pi-\sigma$ and $\pi-\omega$ contributions differ from those of Ref. [14], where $\langle W(\pi-\sigma)\rangle=1.003 \mathrm{MeV}$ and $\langle W(\pi-\omega)\rangle=-0.770 \mathrm{MeV}$ were obtained with a wave function calculated with the Paris $N N$ potential only. This difference is a consequence of the 


\begin{tabular}{|c|c|c|c|c|}
\hline $3 N$ potential & Reid & Paris & Nijmegen 93 & Bonn B \\
\hline$\pi \pi\left(a^{\prime}\right)$ & -0.096 & -0.113 & -0.124 & -0.130 \\
\hline$\pi \pi(b)$ & -1.822 & -1.882 & -2.086 & -1.837 \\
\hline$\pi \pi(d)$ & -0.364 & -0.279 & -0.319 & -0.256 \\
\hline Total $\pi \pi$ & -2.282 & -2.274 & -2.528 & -2.223 \\
\hline$\pi \rho(\mathrm{K} R)$ & 0.055 & 0.063 & 0.097 & 0.113 \\
\hline$\pi \rho\left(\Delta^{+}\right)$ & 0.156 & 0.148 & 0.164 & 0.173 \\
\hline$\pi \rho\left(\Delta^{-}\right)$ & 0.101 & 0.142 & 0.179 & 0.174 \\
\hline$\pi \rho(T)$ & -0.026 & -0.036 & -0.036 & -0.037 \\
\hline Total $\pi \rho$ & 0.287 & 0.317 & 0.403 & 0.424 \\
\hline$\pi \sigma(Z)$ & 0.959 & 1.359 & 1.632 & 2.108 \\
\hline$\pi \sigma\left(N^{*}\right)$ & -0.497 & -0.478 & -0.581 & -0.381 \\
\hline Total $\pi \sigma$ & 0.463 & 0.881 & 1.051 & 1.727 \\
\hline$\pi \omega(Z)$ & -0.902 & -1.422 & -1.722 & -2.377 \\
\hline$\pi \omega\left(N^{*}\right)$ & 0.456 & 0.453 & 0.561 & 0.371 \\
\hline Total $\pi \omega$ & -0.446 & -0.968 & -1.162 & -2.005 \\
\hline Total $\pi \sigma+\pi \omega$ & 0.016 & -0.087 & -0.110 & -0.278 \\
\hline Total $3 N$ potentials & -1.979 & -2.045 & -2.236 & -2.077 \\
\hline
\end{tabular}

TABLE VIII: Expectation values (in $\mathrm{MeV}$ ) of the components of the $3 N$ force in Hamiltonians with different $N N$ potentials, calculated with eigenfunctions of the full Hamiltonian containing all listed $3 N$ potentials.

different coupling constants and cut-off parameters used in Ref. [14]. The results collected in Tables VIII and VI show again that also the short-range $3 N$ forces are non-perturbative: even for the small sum of all $\pi-\sigma$ and $\pi-\omega$ contributions we get, for example with the Paris potential, $-0.048 \mathrm{MeV}$ from Table IV and -0.087 MeV from Table VI.

Finally, in Fig. 2 we represent the dependence of the binding energy for two different $N N$ models as a function of the $\pi N N$ cut-off parameter. The short-range forces do make $E_{t}$ less cut-off dependent, compared to the case when only the $\pi-\pi$ potential is included. But the overall effect of the $\pi-\sigma$ and $\pi-\omega$ potentials is rather small. We emphasize again that 
this sum depends crucially on the values of the coupling constants used in our calculations, in particular on the poorly determined ratio $g_{\sigma}^{*} / g_{\omega}^{*}$.

\section{DETERMINATION OF THE LOW ENERGY CONSTANTS}

\section{A. Effective couplings}

To connect to the $3 N$ force following from ChPT, let us now consider "the pointlike limit", i.e., shrinking the propagators of heavy mesons to a point by taking $m_{B}^{2} \tilde{\Delta}_{B}\left(\mathbf{q}_{3}\right) \rightarrow 1$ ( $B=\rho, \omega, \sigma)$, and deduce the effective contact vertices. In more detail, we separate from the potential two diagrams in which the pion couples to the second nucleon and re-write the corresponding potentials as the product

$$
V_{2}^{a}(\pi)\left(V_{13}^{a}+V_{31}^{a}\right) \quad \text { with } \quad V_{2}^{a}(\pi)=-\frac{g}{2 m} \tau_{2}^{a} \boldsymbol{\sigma}_{2} \cdot \mathbf{q}_{2} \tilde{\Delta}_{\pi}\left(\mathbf{q}_{2}\right),
$$

where the factor $V_{2}^{a}(\pi)$ includes an overall factor $i$, the $\pi N N$ vertex, and the pion propagator. Hence $V_{13}^{a}+V_{31}^{a}$ is the vertex function of the effective point Lagrangian

$$
\mathcal{L}^{\text {point }}=\left(N^{\dagger} \Gamma_{1} N\right)\left(N^{\dagger} \Gamma_{3} N\right) \pi^{a}
$$

The effective leading order Lagrangian is taken in the form

$$
\begin{aligned}
\mathcal{L}^{(1)}= & \mathcal{L}^{(1)}(\alpha)+\mathcal{L}^{(1)}(\beta), \\
\mathcal{L}^{(1)}(\alpha)= & -\alpha_{1}\left(N^{\dagger} N\right)\left(N^{\dagger} \boldsymbol{\sigma} \tau^{a} N\right) \cdot \nabla \pi^{a}-\alpha_{2}\left(N^{\dagger} \boldsymbol{\sigma} N\right)\left(N^{\dagger} \tau^{a} N\right) \cdot \nabla \pi^{a} \\
& -\alpha_{3} \epsilon^{a b c}\left(N^{\dagger} \boldsymbol{\sigma} \tau^{b} N\right) \times\left(N^{\dagger} \boldsymbol{\sigma} \tau^{c} N\right) \cdot \boldsymbol{\nabla} \pi^{a}, \\
\mathcal{L}^{(1)}(\beta)= & +\beta_{1} \pi^{a}\left(N^{\dagger} \boldsymbol{\sigma} \tau^{a} N\right) \cdot \nabla\left(N^{\dagger} N\right)+\beta_{2} \pi^{a}\left(N^{\dagger} \boldsymbol{\sigma} N\right) \cdot \nabla\left(N^{\dagger} \tau^{a} N\right) \\
& +\beta_{3} \epsilon^{a b c} \pi^{a} \epsilon_{k i j}\left(N^{\dagger} \sigma_{i} \tau^{b} N\right) \nabla_{k}\left(N^{\dagger} \sigma_{j} \tau^{c} N\right) .
\end{aligned}
$$

It generalizes the Lagrangian considered in Ref. [10] by including (in $\mathcal{L}^{(1)}(\beta)$ ) vertices with derivatives of nucleon fields. ${ }^{3}$

For the local part of the $\pi-\rho$ potential with a timelike $\rho$-exchange (16) we thus obtain

$$
\mathcal{L}^{\text {point }}(\pi-\rho T, \mathrm{PV}+)=\frac{g g_{\rho}^{2}}{2 m^{2} m_{\rho}^{2}} \pi^{a}\left(N^{\dagger} \boldsymbol{\sigma} N\right) \boldsymbol{\nabla}\left(N^{\dagger} \tau^{a} N\right),
$$

\footnotetext{
${ }^{3}$ In this paper we use the leading order $\pi N N$ coupling with the plus $\operatorname{sign}\left(\mathcal{L}_{\pi N N}=+\frac{g}{2 m}\left(N^{\dagger} \boldsymbol{\sigma} \tau^{a} N\right) \cdot \nabla \pi^{a}\right)$, in agreement with Hüber et al. 9, 15], but opposite to Epelbaum et al. 10]. Therefore, the Lagrangian (35) has the opposite sign compared to Eq.(2.3) of Ref. [10].
} 
Paris potential with three-nucleon force

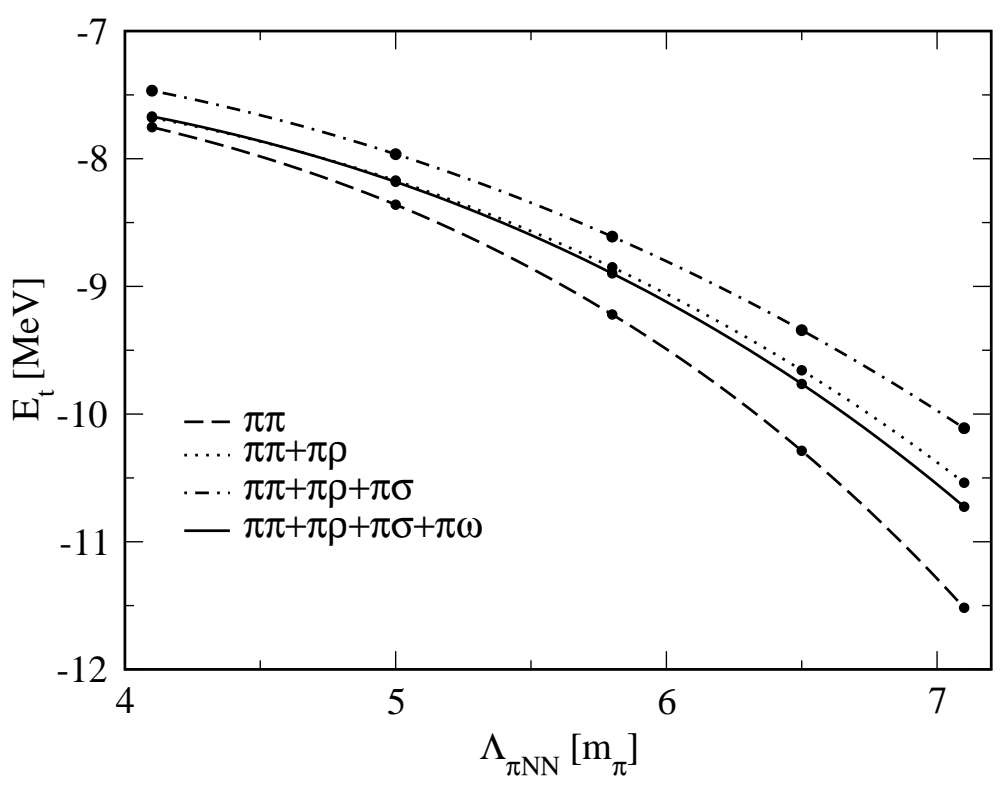

Nijmegen 93 potential with three-nucleon force

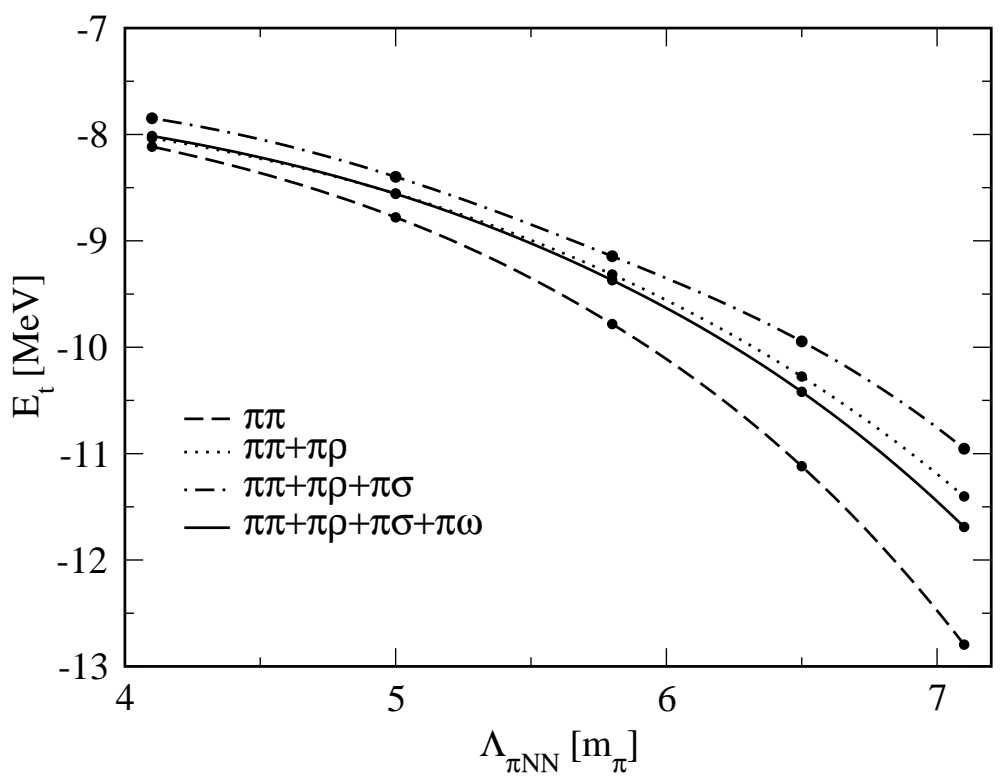

FIG. 2: Dependence of the triton binding energy on the $\pi N N$ cut-off parameter, calculated for Hamiltonians with the Paris and the Nijmegen $93 N N$ potentials and various contributions to the $3 N$ force, which are successively added in the following way: $\pi-\pi$ exchange (dashed line), plus $\pi-\rho$ (dotted line), plus $\pi-\sigma$ (dashed-dotted line), plus $\pi-\omega$ (solid line). The actual calculations are indicated by the full circles, the lines are drawn to guide the eye. 
and comparing to (36) we extract

$$
\beta_{2}(\pi-\rho T, \mathrm{PV}+)=\frac{g g_{\rho}^{2}}{2 m^{2} m_{\rho}^{2}},
$$

where for PS coupling there would be an additional factor $1+\kappa_{\rho}$. For the local part of the $\pi-\rho \mathrm{KR}$ term (19) one gets

$$
\begin{aligned}
\mathcal{L}^{\text {point }}(\pi-\rho S, \mathrm{KR}) & =-\frac{g g_{\rho}^{2}\left(1+\kappa_{\rho}\right)}{2 m^{2} m_{\rho}^{2}} \epsilon_{a b c} \epsilon_{k i j} \pi^{a}\left(N^{\dagger} \sigma_{i} \tau^{b} N\right) \nabla_{k}\left(N^{\dagger} \sigma_{j} \tau^{c} N\right) . \\
\beta_{3}(\pi-\rho S, \mathrm{KR}) & =-\frac{g g_{\rho}^{2}\left(1+\kappa_{\rho}\right)}{2 m^{2} m_{\rho}^{2}}
\end{aligned}
$$

For the Born $\pi-\sigma$ exchange the point low-energy Lagrangians corresponding to the limit of (21) and (22) are

$$
\begin{aligned}
\mathcal{L}^{\text {point }}(\pi-\sigma, \mathrm{PV}+) & =-\frac{g g_{\sigma}^{2}}{2 m^{2} m_{\sigma}^{2}} \pi^{a}\left(N^{\dagger} \tau^{a} \boldsymbol{\sigma} N\right) \cdot \boldsymbol{\nabla}\left(N^{\dagger} N\right), \\
\beta_{1}(\pi-\sigma, \mathrm{PV}+) & =-\frac{g g_{\sigma}^{2}}{2 m^{2} m_{\sigma}^{2}}, \\
\mathcal{L}^{\text {point }}(\pi-\sigma, \mathrm{PS}-) & =+\frac{g g_{\sigma}^{2}}{2 m^{2} m_{\sigma}^{2}}\left(N^{\dagger} N\right)\left(N^{\dagger} \boldsymbol{\sigma} \tau^{a} N\right) \cdot\left(\boldsymbol{\nabla} \pi^{a}\right), \\
\alpha_{1}(\pi-\sigma, \mathrm{PS}-) & =-\frac{g g_{\sigma}^{2}}{2 m^{2} m_{\sigma}^{2}} .
\end{aligned}
$$

For $\pi-\omega$ exchange we obtain

$$
\begin{aligned}
\mathcal{L}^{\text {point }}(\pi-\omega, \text { PS- }) & =+\frac{g g_{\omega}^{2}}{2 m^{2} m_{\omega}^{2}} \pi^{a}\left(N^{\dagger} \boldsymbol{\sigma} \tau^{a} N\right) \cdot \nabla\left(N^{\dagger} N\right), \\
\beta_{1}(\pi-\omega, \text { PS- }) & =+\frac{g g_{\omega}^{2}}{2 m^{2} m_{\omega}^{2}} .
\end{aligned}
$$

The corresponding effective interaction for the PV+ term includes an additional factor $1+\kappa_{\omega} \approx 1$, which we will neglect in the following. For the contributions with an intermediate $N^{*}$ (note that the $N^{*}$ propagator is already used in static point approximation) one derives

$$
\begin{aligned}
\mathcal{L}^{\text {point }}\left((\pi-(\sigma+\omega)), \mathrm{N}^{*}\right) & =\frac{g^{*}}{m\left(m^{*}-m\right)}\left(\frac{g_{\sigma} g_{\sigma}^{*}}{m_{\sigma}^{2}}-\frac{g_{\omega} g_{\omega}^{*}}{m_{\omega}^{2}}\right)\left(N^{\dagger} N\right)\left(N^{\dagger} \boldsymbol{\sigma} \tau^{a} N\right) \cdot \nabla \pi^{a}, \\
\alpha_{1}\left(\sigma+\omega, \mathrm{N}^{*}\right) & =-\frac{g^{*}}{m\left(m^{*}-m\right)}\left(\frac{g_{\sigma} g_{\sigma}^{*}}{m_{\sigma}^{2}}-\frac{g_{\omega} g_{\omega}^{*}}{m_{\omega}^{2}}\right) .
\end{aligned}
$$

It is interesting that for $\pi-\sigma$ exchange the result with PS $\pi N N$ coupling is closer to the form suggested in Refs. [10, 15]. Note also that the difference between the Lagrangians (41) 
and (43) (we label it "cont" since it corresponds to the chiral contact term connecting PV and PS couplings) is equivalent to

$$
\mathcal{L}^{\text {point }}(\pi-\sigma, \text { cont })=\frac{g g_{\sigma}^{2}}{2 m^{2} m_{\sigma}^{2}}\left(N^{\dagger} N\right)\left[\left(\boldsymbol{\nabla} N^{\dagger}\right) \cdot \boldsymbol{\sigma} \tau^{a} \pi^{a} N+N^{\dagger} \tau^{a} \pi^{a} \boldsymbol{\sigma} \cdot(\boldsymbol{\nabla} N)\right],
$$

This interaction is exactly the same as the second term in (2) (with $c \rightarrow-g g_{\sigma}^{2} /\left(2 m m_{\sigma}^{2}\right)$ ). Note that the Lagrangian (2) follows from the re-definition of the pion field (11), while the so-called chiral rotation (which transforms in lowest order from $\pi N N$ PV to PS coupling) is a redefinition of the nucleon field. It is argued in [15] that the interactions in (2) are unnatural from the point of view of ChPT and should be discarded. The appearance of such a term among the effective contact interactions raises the suspicion that the dynamical model (conforming with chiral symmetry) with a $\sigma$ meson and PV $\pi N N$ coupling contains also the mechanism corresponding to the first term in (2) and that, in analogy to the discussion in [15], both should be discarded. Let us also point out that the most natural way of introducing the $\sigma$ meson into a chirally symmetric Lagrangian is the linear $\sigma$ model with PS $\pi N N$ coupling (which, however, fixes the coupling constant $g_{\sigma}$ ).

\section{B. Numerical results and comparison to ChPT}

In Table $\mathrm{X}$ we list the numerical values for the dimensionless low-energy constants

$$
\begin{gathered}
\tilde{\alpha}_{i}=4 f_{\pi}^{3} \Lambda_{\chi} \alpha_{i}, \\
\tilde{\beta}_{i}=4 f_{\pi}^{3} \Lambda_{\chi} \beta_{i},
\end{gathered}
$$

where $\Lambda_{\chi}=700 \mathrm{MeV}$ as in Ref. [10]. The constants $\tilde{\alpha}_{i}, \tilde{\beta}_{i}$ following from the considered heavy meson exchanges appear to be of natural size. Notice that - with the exception of the potentials with $N^{*}$ and the $\pi-\sigma$ Born term (the latter only with PS $\pi N N$ coupling) all effective vertices are of the form (36), i.e., they involve derivatives of nucleon fields. In particular, we do not get any contributions to the effective couplings $\tilde{\alpha}_{2}$ and $\tilde{\alpha}_{3}$.

There is a pronounced cancellation between $\omega$ and $\sigma$ exchanges in the low energy limit, both for the Born (with PV coupling) and Roper contributions. Although the $\omega$ and $\sigma$ terms would always have opposite signs, their sum is rather sensitive to the poorly determined values of the coupling constants. In particular, the almost perfect cancellation of the respective Roper terms appears to be accidental. The timelike part of the $\rho$ exchange is 
rather small for PV $\pi N N$ coupling, but for PS coupling the large factor $1+\kappa_{\rho}$ increases the corresponding $\tilde{\beta}_{2}$ to the order of all the other contributions. These results are completely in line with our results for the corresponding potentials included in their full form into the Faddeev equations, i.e., the low energy limit changes of course the size of the individual contributions, but not their signs and relative magnitude.

The chiral $3 N$ forces and their effect on the $3 N$ observables were studied in Ref. [9] and more recently in Ref. 10]. Unfortunately, the point limit of the short-range forces considered above cannot be compared directly to their results, because neither of these papers includes terms with derivatives of nucleon fields in the Lagrangian. In Ref. [10] it is claimed that these terms can be reduced to the form of (35) through integration by parts. To transform our operators into a form consistent with the effective Lagrangian of Ref. [9], we make the following momentum replacement: $\mathbf{q}_{3} \rightarrow-\mathbf{q}_{2}$. It corresponds to integrating the effective Lagrangians with nucleon derivatives by parts (this is equivalent to the use of momentum conservation at corresponding vertices, $\mathbf{q}_{3}=-\mathbf{q}_{2}-\mathbf{q}_{1}$ ) and keeping only the resultant terms with a pion field derivative (momentum $\mathbf{q}_{2}$ ), while omitting at the same time still another term in the Lagrangian with nucleon field derivatives (leading to the momentum $\mathbf{q}_{1}$ ).

However, our numerical calculations do not justify neglecting the effective Lagrangians with nucleon field derivatives. This is seen clearly from Table $\amalg$ where we compare the effect of momentum replacement in matrix elements (with the fully correlated wavefunction) of the original potentials, their point limits. Since these numbers are quite different, we checked in addition whether the disagreement is due to high momentum components of the wave function by multiplying the operators by the overall exponential form factor of Ref. [10]. However, we do not see any improvement with respect to the agreement between $\langle W\rangle$ and $\left\langle W_{\text {repl }}\right\rangle$.

Nevertheless, since no other numerical estimates of LECs are currently available, we use the momentum replacement prescription to see how the LECs derived from the potentials of this paper compare with those deduced in Refs. [9, 10]. For the LECs the momentum replacement translates into $\beta_{i} \rightarrow \alpha_{i}$.

Hüber et al. [9] introduce two LECs $c_{1}$ and $c_{2}$, related to our $\tilde{\alpha}_{1}$ and $\tilde{\alpha}_{3}$ as follows

$$
\begin{aligned}
& c_{1}=\frac{\Lambda}{4 \Lambda_{\chi}} \tilde{\alpha}_{1}=\frac{5}{14} \tilde{\alpha}_{1} \\
& c_{2}=\frac{\Lambda}{2 \Lambda_{\chi}} \tilde{\alpha}_{3}=\frac{10}{14} \tilde{\alpha}_{3},
\end{aligned}
$$




\begin{tabular}{lcccr}
\hline \hline & \multicolumn{2}{c}{ Paris } & \multicolumn{2}{c}{ Nijmegen 93} \\
$3 N$ potential $W$ & $\langle W\rangle$ & $\left\langle W_{\text {repl }}\right\rangle$ & $\langle W\rangle$ & $\left\langle W_{\text {repl }}\right\rangle$ \\
\hline$\pi-\rho(\mathrm{KR})$ & 0.063 & -0.220 & 0.097 & -0.231 \\
$\pi-\rho(\mathrm{KR})$ point limit & 0.045 & -0.223 & 0.085 & -0.250 \\
$\pi-\rho(\mathrm{KR})$ point limit $\times$ exp. ff. & 0.053 & 0.064 & 0.094 & 0.154 \\
\hline$\pi-\sigma(\mathrm{Z})$ & 1.359 & -1.329 & 1.632 & -1.617 \\
$\pi-\sigma(\mathrm{Z})$ point limit & 0.873 & -0.531 & 0.921 & -0.693 \\
$\pi-\sigma(\mathrm{Z})$ point limit $\times$ exp. ff. & 2.034 & -0.015 & 2.409 & 0.186 \\
\hline$\pi-\rho(\mathrm{T})$ & -0.036 & -0.012 & -0.036 & -0.016 \\
$\pi-\rho(\mathrm{T})$ point limit & -0.050 & -0.016 & -0.049 & -0.020 \\
$\pi-\rho(\mathrm{T})$ point limit $\times$ exp. ff. & -0.027 & 0.004 & -0.024 & 0.011 \\
\hline \hline
\end{tabular}

TABLE IX: Validity of the momentum replacement prescription. Matrix elements (in MeV) of $3 N$ forces related by the momentum replacement prescription described in the text are compared. For each of the three considered cases also the point limit, as well as the point limit multiplied with an additional exponential form factor are shown. The matrix elements are calculated with a wave function corresponding the Paris $N N$ potential together with the $3 N$ forces of Table VIII

with $\Lambda=1 \mathrm{GeV}$. They fit these constants to $A_{y}$ from $n d$ scattering, arguing that $c_{1}$ is fixed to about $c_{1} \sim-3$ (from $A_{y}$ at $3 \mathrm{MeV}$ ) and $c_{2} \sim 0.5 \ldots 1.0$ (from $A_{y}$ at $10 \mathrm{MeV}$ ). From the longshort range potentials considered in this paper, only the $\mathrm{KR} \pi-\rho$ potential contributes in the low-energy limit to the constant $c_{2}$, yielding $c_{2}=-1.55$ (see Table $\underline{\mathrm{X}}$ ) which differs form the value cited above both in sign and magnitude. However, it should be stressed once more that the momentum replacement was used in deriving $c_{2}(K R)$ to convert the corresponding point-like vertex from the form (36) to the form (35). The total $c_{1}$ from Table $\mathrm{X}$ is $c_{1} \sim 1$, again quite different from that of Ref. [9]. Here it may be worth mentioning that in Ref. [9] the second term in the Lagrangian (35) proportional to $\alpha_{2}$ is not considered.

From this comparison of LECs it is at the moment hard to see whether the potentials used in this paper could provide a reasonable description of $A_{y}$ (and if there is therefore a reason to believe that we have not missed some other important short-range effects). The comparison of LECs is seriously hampered by the fact that the ChPT derivation of Refs. 


\begin{tabular}{lrr}
\hline \hline Diagram & LECs & $c_{i}$ \\
\hline$\rho-T(P V)$ & $\tilde{\beta}_{2}=0.29$ & \\
$\rho-T(P S)$ & $\tilde{\beta}_{2}=2.17$ & \\
$\rho-S(K R)$ & $\tilde{\beta}_{3}=-2.17$ & $c_{2}(\mathrm{repl})=-1.55$ \\
\hline$\omega-\operatorname{Born}$ & $\tilde{\beta}_{1}=8.32$ & $c_{1}(\mathrm{repl})=2.97$ \\
$\sigma-\operatorname{Born}(P V)$ & $\tilde{\beta}_{1}=-6.29$ & $c_{1}(\mathrm{repl})=-2.25$ \\
$\sigma-\operatorname{Born}(P S)$ & $\tilde{\alpha}_{1}=-6.29$ & $c_{1}=-2.25$ \\
\hline$\omega-N^{*}$ & $\tilde{\alpha}_{1}=2.99$ & $c_{1}=1.07$ \\
$\sigma-N^{*}$ & $\tilde{\alpha}_{1}=-2.26$ & $c_{1}=-0.81$ \\
\hline \hline
\end{tabular}

TABLE X: Dimensionless low-energy constants defined by Eqs. (50151), derived from $\pi-\rho, \pi-\omega$ and $\pi-\sigma$ exchanges using the masses and coupling constants of Table (III). The constants $c_{i}($ repl) were obtained with the help of the momentum replacement, for $\sigma-$ Born term this replacement is needed only if PV $\pi N N$ coupling is used

9], 10] does not consider effective operators to which the meson-exchange potentials reduce to in the low-energy limit.

In Ref. 10] it is further argued that the Lagrangian (35) reduces effectively to just one independent term when used with $3 N$ wave functions which are antisymmetric in the spin and isospin subspace of the two nucleons coupled to the contact vertex. Hence only one effective coupling constant (called $D$ ) for the $\pi N N N N$ vertex is considered in their numerical analysis. Assuming this, the matrix element of the vertex function corresponding to the Lagrangian (35) is reduced to the matrix element of only one particular combination of spin-isospin operators. Indeed, for

$$
v_{13}^{a}=\left[\alpha_{1}\left(\tau_{1}^{a} \boldsymbol{\sigma}_{1}+\tau_{3}^{a} \boldsymbol{\sigma}_{3}\right)+\alpha_{2}\left(\tau_{1}^{a} \boldsymbol{\sigma}_{3}+\tau_{3}^{a} \boldsymbol{\sigma}_{1}\right)+2 \alpha_{3}\left(\boldsymbol{\tau}_{1} \times \boldsymbol{\tau}_{3}\right)^{a}\left(\boldsymbol{\sigma}_{1} \times \boldsymbol{\sigma}_{3}\right)\right],
$$

it holds that

$$
A_{13} v_{13}^{a}=\frac{\alpha_{1}-\alpha_{2}+4 \alpha_{3}}{4}\left[\left(\boldsymbol{\tau}_{1}-\boldsymbol{\tau}_{3}\right)^{a}\left(\boldsymbol{\sigma}_{1}-\boldsymbol{\sigma}_{3}\right)-\left(\boldsymbol{\tau}_{1} \times \boldsymbol{\tau}_{3}\right)^{a}\left(\boldsymbol{\sigma}_{1} \times \boldsymbol{\sigma}_{3}\right)\right]
$$

where $A_{13}=\frac{1}{2}\left(1-P_{13}^{\tau} P_{13}^{\sigma}\right)$ is the spin-isospin antisymmetrization operator for particles 1 and 3. Therefore, if only such components of $3 N$ wave functions are important, the effect 
of (35) can be represented by a single term. However, we would like to point out that the $3 N$ wave function contains also components which are symmetric in spin-isospin space (and antisymmetric in their spatial part). For such components one gets instead of (55)

$$
\begin{aligned}
S_{13} v_{13}^{a}= & \frac{\alpha_{1}+\alpha_{2}}{2}\left(\boldsymbol{\tau}_{1}+\boldsymbol{\tau}_{3}\right)^{a}\left(\boldsymbol{\sigma}_{1}+\boldsymbol{\sigma}_{3}\right) \\
& +\frac{\alpha_{1}-\alpha_{2}-4 \alpha_{3}}{4}\left[\left(\boldsymbol{\tau}_{1}-\boldsymbol{\tau}_{3}\right)^{a}\left(\boldsymbol{\sigma}_{1}-\boldsymbol{\sigma}_{3}\right)+\left(\boldsymbol{\tau}_{1} \times \boldsymbol{\tau}_{3}\right)^{a}\left(\boldsymbol{\sigma}_{1} \times \boldsymbol{\sigma}_{3}\right)\right],
\end{aligned}
$$

where $S_{13}=\frac{1}{2}\left(1+P_{13}^{\tau} P_{13}^{\sigma}\right)$ is the spin-isospin symmetrization operator for particles 1 and 3 . If these components are not omitted, the matrix elements of the potentials derived from the $\pi N N N N$ interaction (35) still contain three different combinations of the constants $\alpha_{1}, \alpha_{2}$, and $\alpha_{3}$. The components of the triton wave function with odd orbital angular momentum between nucleons 1 and 3 contribute about 5 per cent to the total norm. Even though the weight of these states in the norm is not large, their effect can become significant in matrix elements of operators with the proper tensor structure. Moreover, $N N$ P-waves are known to very important for the description of $A_{y}$ in $N d$ scattering.

If we neglect for the moment the symmetric spin-isospin wave function components and interactions with nucleon field derivatives, then the effect of the $\alpha_{i}$ terms is reduced to the calculation of the matrix element of (55). In this approximation, the dimensionless constant $c_{D}$ of Ref. [10] is given in terms of $\tilde{\alpha}_{i}$ as

$$
c_{D}=-\tilde{\alpha}_{1}+\tilde{\alpha}_{2}-4 \tilde{\alpha}_{3}
$$

From Table $\mathrm{X}$ we get $c_{D} \sim 6 \ldots 8$, depending on the type of the $\pi N N$ coupling. This numerical value of $c_{D}$ is larger than $c_{D} \sim 1.8 \ldots 3.6$ obtained in Ref. [10]. In fact, the KR contribution dominates our value of $c_{D}$, due to the factor 4 in the last term of (57)). As in the comparisons above to Ref. [9], we had to compare quite different effective Lagrangians and the approximations made in establishing the correspondence between the effective constants appear to be too crude to allow drawing clear conclusions.

Finally we note that the efforts of determining the LECs in 9] may have resulted in a better description of $A_{y}$ with different constants, had the additional freedom in the parameters of the TM $\pi-\pi$ force due to the experimental uncertainties discussed in Sec. IIIA been taken into account. 


\section{CONCLUSIONS}

The conclusions of this paper can be summarized in three points:

1. When considering the effect of the TM $\pi-\pi$ exchange $3 N$ force on the triton binding energy, only the variation of the cut-off parameter, which is often adjusted to reproduce the experimental value, is studied in the literature.

However, the $\pi-\pi$ force contains the $\pi \mathrm{N}$ scattering amplitude as a building block, which at low pion momenta has a model independent form parameterized by three constants which have to be extracted from experiment. In contrast to the cut-off parameter, each of these three constants multiply different spin-isospin operators, and therefore act differently on the various channels of the wavefunction. We calculate the propagation of the experimental errors of the $\pi N$ data, which are used to extract the off-shell TM $\pi N$ scattering amplitude, into the $3 N$ force parameters. As a consequence, the triton binding energy calculated with the TM $\pi-\pi$ potential has an uncertainty of about $\pm 0.4 \mathrm{MeV}$, which is almost entirely due to the experimental errors in the nucleon sigma term.

2. The long-short range $\pi-\sigma$ and $\pi-\omega$ exchange $3 N$ forces individually have large effects on the triton binding, but two kinds of cancellations determine their net effect. We find contributions of opposite sign and comparable or very close in magnitude from $Z$-graph and $N^{*}$ excitation, respectively, in the $\pi-\sigma$ and $\pi-\omega 3 N$ forces. Also $Z$-graph and $N^{*}$ potentials cancel in part for each of these long-short range forces separately.

The extent of the cancellation is controlled decisively by the ratio between the $\sigma N^{*}(1440) N$ and $\omega N^{*}(1440) N$ coupling constants. If this ratio is taken to be the same as for the $\sigma N N$ and $\omega N N$ coupling constants, as suggested by the naive constituent quark model, the attraction of the $\omega$ exchange, originated by the Born terms of relativistic order, dominates.

The change in binding energy caused by these $3 N$ potentials cannot be calculated perturbatively, since their inclusion in the Hamiltonian changes the wavefunction significantly. For instance, we find that, although the net effect of $\pi-\sigma$ and $\pi-\omega$ exchange $3 N$ forces on the binding energy is small, the resulting wavefunction yields rather different matrix elements of individual interactions from the ones calculated without those terms in the Hamiltonian. Therefore, it would be very interesting to study these variations in the wavefunction, for

instance in electron scattering on the $3 N$ bound state. Furthermore, these potentials may 
strongly influence low-energy $N d$ scattering observables.

In the case of PS $\pi N N$ coupling, both $Z$-diagrams are attractive, resulting in a strong overbinding of the triton. In the future, these $Z$-diagrams should be complemented by additional potentials derived from chiral contact $\pi N N \sigma$ and $\pi N N \omega$ vertices.

3. We extracted the LECs from the low-energy limit of the long-short range meson exchange potentials and compared with the ChPT results of Refs. [9, 10]. The constants we obtain are of natural size. Unfortunately, a direct comparison with the ChPT results is not possible since we arrive at contact vertices of different structure. To translate them into the ChPT form certain approximations are necessary, the validity of which we found to be unfounded in our numerical evaluation. It does not come as a surprise that our LECs do not agree with those of Refs. 9, 10]. Efforts should be joined from the two sides (meson-exchange description and ChPT methods) to investigate this question further.

\section{Acknowledgments}

J.A. was supported by the grants GA CR 202/00/1669, GA CR 202/03/0210 and by the Lisbon project. He would like to thank his Portuguese colleagues for the warm hospitability during his stay in Lisbon. M.T.P. and A.S. were supported by FCT and FEDER under grants CERN/FIS/43709/2001 and POCTI/FNU/40834/2001.

\section{APPENDIX A: CONTRIBUTIONS OF NUCLEON BORN DIAGRAMS}

We will give here some details of the derivation of nucleon Born diagram contributions to the $3 N$ force from the exchange of one pion and one heavy meson $(B)$, since we experienced some misunderstandings and many questions when discussing their origin. Our treatment is a condensed version of the technique developed in a number of papers on e.m. meson exchange currents [35, 36, 37]. We pay special attention to the dependence of our results on the type of the $\pi N N$ vertex.

The generic Feynman amplitude $\mathcal{W}(1)$ corresponding to Fig. 17 reads:

$$
\mathcal{W}(1)=\mathcal{F}^{a} \mathcal{D}^{a}+2 \leftrightarrow 3
$$

where we gather all factor connected with the "active" first nucleon into the amplitude $\mathcal{F}$ 
and the rest into the amplitude $\mathcal{D}$

$$
\begin{aligned}
& \mathcal{D}^{a}=-\bar{u}\left(\mathbf{p}_{2}^{\prime}\right) \Gamma^{a}\left(\pi, q_{2}\right) u\left(\mathbf{p}_{2}\right) \bar{u}\left(\mathbf{p}_{3}^{\prime}\right) \Gamma\left(B, q_{3}\right) u\left(\mathbf{p}_{3}\right) \Delta_{\pi}\left(q_{2}\right) \Delta_{B}\left(q_{3}\right), \\
& \mathcal{F}^{a}=\bar{u}\left(\mathbf{p}_{1}^{\prime}\right)\left[\Gamma^{a}\left(\pi,-q_{2}\right) S\left(P^{\prime}\right) \Gamma\left(B,-q_{3}\right)+\Gamma\left(B,-q_{3}\right) S(P) \Gamma^{a}\left(\pi,-q_{2}\right)\right] u\left(\mathbf{p}_{1}\right),
\end{aligned}
$$

where $S(p)=\left(i \gamma_{\mu} p_{\mu}+m\right)^{-1}$ and $\Delta_{B}(q)=\left(m_{B}^{2}+q^{2}\right)^{-1}$ (the Pauli metric is used), $P=$ $p_{1}^{\prime}+q_{3}=p_{1}-q_{2}, P^{\prime}=p_{1}-q_{3}=p_{1}^{\prime}+q_{2}$ with $q_{i}=p_{i}^{\prime}-p_{i}$, and we suppress (unless we specify $B$ ) additional isospin and Lorentz indices, that would appear for non-scalar and charged meson $B$. The pion $(\pi N N)$ vertex functions (corresponding to $i \mathcal{L}$ ) are

$$
\begin{aligned}
\Gamma^{a}(\pi-\mathrm{PS}) & =-g \gamma_{5} \tau^{a}, \\
\Gamma^{a}(\pi-\mathrm{PV}, q) & =i \frac{g}{2 m} q_{\mu} \gamma_{\mu} \gamma_{5} \tau^{a},
\end{aligned}
$$

where $q=p^{\prime}-p$ is the momentum of the pion entering the vertex (the signs of our vertex functions differ from those of Ref. [37], we adopted the current convention to agree with that usually used in ChPT calculations). The vertex functions $\Gamma(B, q)$ for the heavy mesons $B$ will be specified later. Note, that from the identities

$$
\begin{aligned}
\bar{u}\left(\mathbf{p}_{1}^{\prime}\right) \Gamma^{a}\left(\pi-\mathrm{PV},-q_{2}\right) S\left(P^{\prime}\right) & =\bar{u}\left(\mathbf{p}_{1}^{\prime}\right)\left[\Gamma^{a}(\pi-\mathrm{PS}) S\left(P^{\prime}\right)+\frac{g}{2 m} \gamma_{5} \tau_{1}^{a}\right] \\
S(P) \Gamma^{a}\left(\pi-\mathrm{PV},-q_{2}\right) u\left(\mathbf{p}_{1}\right) & =\left[S(P) \Gamma^{a}(\pi-\mathrm{PS})+\frac{g}{2 m} \gamma_{5} \tau_{1}^{a}\right] u\left(\mathbf{p}_{1}\right),
\end{aligned}
$$

it follows

$$
\begin{aligned}
\mathcal{F}_{\mathrm{PV}}^{a} & =\mathcal{F}_{\mathrm{PS}}^{a}+\mathcal{F}_{\mathrm{cont}}^{a}, \\
\mathcal{F}_{\mathrm{cont}}^{a} & =\frac{g}{2 m} \bar{u}\left(\mathbf{p}_{1}^{\prime}\right)\left\{\gamma_{5} \tau_{1}^{a}, \Gamma\left(B,-q_{3}\right)\right\}_{+} u\left(\mathbf{p}_{1}\right)
\end{aligned}
$$

The "contact" amplitude arises from the usual chiral contact interaction, which appears in the chiral rotation from the $\mathrm{PV}$ to $\mathrm{PV} \pi N N$ coupling.

To get the quantum-mechanical $3 N$ potential from the Feynman amplitude (A1), it is necessary to subtract the part which is in the quantum mechanical description already included in the $T$ matrix in the iterations of the $N N$ potential. We split the nucleon propagator into its positive and negative energy parts $S(p)=S^{+}(p)+S^{-}(p)$ :

$$
S(p)=\frac{m-i \gamma_{\mu} p_{\mu}}{p^{2}+m^{2}}=\frac{m+E \gamma_{4}-i \boldsymbol{\gamma} \cdot \mathbf{p}}{2 E\left(E-p_{0}\right)}+\frac{m-E \gamma_{4}-i \boldsymbol{\gamma} \cdot \mathbf{p}}{2 E\left(E+p_{0}\right)}=\frac{u(\mathbf{p}) \bar{u}(\mathbf{p})}{E-p_{0}}-\frac{v(-\mathbf{p}) \bar{v}(-\mathbf{p})}{E+p_{0}},
$$


(where $E^{2}=\mathbf{p}^{2}+m^{2}$ ) and define the corresponding amplitudes $\mathcal{W}^{ \pm}(1)$ and $\mathcal{F}^{ \pm}$. The spinors $u$ and $v$ are given by:

$$
u(\mathbf{p})=\sqrt{\frac{E+m}{2 E}}\left(\begin{array}{c}
1 \\
\frac{\boldsymbol{\sigma} \cdot \mathbf{p}}{E+m}
\end{array}\right) ; \quad v(-\mathbf{p})=\sqrt{\frac{E+m}{2 E}}\left(\begin{array}{c}
-\frac{\boldsymbol{\sigma} \cdot \mathbf{p}}{E+m} \\
1
\end{array}\right) .
$$

For the calculations of this paper it is sufficient to keep only the leading order in $p / m$ and replace $E \rightarrow m$.

The true "pair" (or "Z-diagram") contributions to the $3 N$ potential are defined by the straightforward non-relativistic reduction (i.e. the decomposition of the spinor matrix elements in powers of $p / m$ keeping only leading order terms) of the $\mathcal{W}^{-}(1)$. In the order considered, this contribution is non-zero only for the PS $\pi N N$ coupling for any meson exchange. For this coupling (and again, at the given order in $p / m) \mathcal{W}^{+}(1)$ with positive energy nucleon in the intermediate state corresponds to the iteration of the lowest order non-relativistic OPEP potential (which is independent of the type of $\pi N N$ coupling). On the other hand, for the PV $\pi N N$ coupling, the pair diagrams $\mathcal{W}^{-}(1)$ do not contribute. But the positive energy part $\mathcal{W}^{+}(1)$ does not exactly equal to the iteration of the non-relativistic OPEP, since in the Feynman amplitude the vertex function is off-mass-shell $\left(P^{2} \neq m^{2} \neq P^{\prime 2}\right)$ while in the iteration of the OPEP this potential is off-energy-shell. One can re-arrange the energies in $q_{20}$ entering the PV vertex function in (A1A3) identically

$$
\begin{aligned}
& q_{20}=P_{0}^{\prime}-E\left(p_{1}^{\prime}\right)=\left(E\left(P^{\prime}\right)-E\left(p_{1}^{\prime}\right)\right)-\left(E\left(P^{\prime}\right)-P_{0}^{\prime}\right), \\
& q_{20}=E\left(p_{1}\right)-P_{0}=\left(E\left(p_{1}\right)-E(P)\right)+\left(E(P)-P_{0}\right) .
\end{aligned}
$$

The first energy differences on the r.h.s. put the PV vertex on its mass shell. The corresponding part of $\mathcal{W}^{+}(1)$ is then again identified with the iteration of the non-relativistic OPEP and it is identical to the full $\mathcal{W}^{+}(1)$ for the PS coupling. But the second terms on $q_{20}$ cancel the denominator of $S^{+}\left(P^{\prime}\right)$ or $S^{+}(P)$ and give rise to a contribution to the quantum mechanical potential $W^{+}(1)$.

To sum it up, the relevant contributions to the $3 N$ potential are

$$
\begin{aligned}
W(1) & =F^{a} D^{a}+2 \leftrightarrow 3, \\
D^{a} & \approx \frac{g}{2 m} \tau_{2}^{a}\left(\boldsymbol{\sigma}_{2} \cdot \mathbf{q}_{2}\right) \bar{u}\left(\mathbf{p}_{3}^{\prime}\right) \Gamma\left(B, q_{3}\right) u\left(\mathbf{p}_{3}\right) \Delta_{\pi}\left(q_{2}\right) \Delta_{B}\left(q_{3}\right), \\
F_{\mathrm{PS}}^{a,-} & \approx \frac{g}{2 m}\left[\bar{u}\left(\mathbf{p}_{1}^{\prime}\right) \gamma_{5} \tau_{1}^{a} v\left(-\mathbf{P}^{\prime}\right) \bar{v}\left(-\mathbf{P}^{\prime}\right) \Gamma\left(B,-q_{3}\right) u\left(\mathbf{p}_{1}\right)+\right.
\end{aligned}
$$




$$
\begin{array}{r}
\left.\bar{u}\left(\mathbf{p}_{1}^{\prime}\right) \Gamma\left(B,-q_{3}\right) v(-\mathbf{P}) \bar{v}(-\mathbf{P}) \gamma_{5} \tau_{1}^{a} u\left(\mathbf{p}_{1}\right)\right] \\
F_{\mathrm{PV}}^{a,+} \approx-\frac{g}{2 m}\left[\bar{u}\left(\mathbf{p}_{1}^{\prime}\right) \gamma_{4} \gamma_{5} \tau_{1}^{a} u\left(\mathbf{P}^{\prime}\right) \bar{u}\left(\mathbf{P}^{\prime}\right) \Gamma\left(B,-q_{3}\right) u\left(\mathbf{p}_{1}\right)-\right. \\
\left.\bar{u}\left(\mathbf{p}_{1}^{\prime}\right) \Gamma\left(B,-q_{3}\right) u(\mathbf{P}) \bar{u}(\mathbf{P}) \gamma_{4} \gamma_{5} \tau_{1}^{a} u\left(\mathbf{p}_{1}\right)\right] \\
F_{\mathrm{cont}}^{a} \approx \frac{g}{2 m} \bar{u}\left(\mathbf{p}_{1}^{\prime}\right)\left\{\gamma_{5} \tau_{1}^{a}, \Gamma\left(B,-q_{3}\right)\right\}_{+} u\left(\mathbf{p}_{1}\right) .
\end{array}
$$

where $\approx$ stands for the non-relativistic reduction. Since only $F^{a,-}$ survives for PS coupling and only $F^{a,+}$ contributes for the PV one (and since the iterations of non-relativistic OPEP are identical in both cases), one gets from (A9)

$$
F_{\mathrm{PV}}^{a,+}=F_{\mathrm{PS}}^{a,-}+F_{\mathrm{cont}}^{a},
$$

and therefore

$$
W_{\mathrm{PV}}^{+}=W_{\mathrm{PS}}^{-}+W_{\text {cont }}
$$

for all heavy meson exchanges considered.

It remains to list the vertex functions for the $\sigma, \omega$ and $\rho$ mesons and the results of the non-relativistic reduction. For the scalar isoscalar $\sigma$ meson one gets

$$
\begin{aligned}
\Gamma(\sigma) & =i g_{\sigma}, \\
D^{a}(\sigma) & =+i \frac{g g_{\sigma}}{2 m} \tau_{2}^{a}\left(\boldsymbol{\sigma}_{2} \cdot \mathbf{q}_{2}\right) \Delta_{\pi}\left(q_{2}\right) \Delta_{\sigma}\left(q_{3}\right), \\
F_{\mathrm{PV}}^{a,+}(\sigma) & =-i \frac{g g_{\sigma}}{2 m^{2}} \tau_{1}^{a}\left(\boldsymbol{\sigma}_{1} \cdot \mathbf{q}_{3}\right), \\
F_{\mathrm{PS}}^{a,-}(\sigma) & =+i \frac{g g_{\sigma}}{2 m^{2}} \tau_{1}^{a}\left(\boldsymbol{\sigma}_{1} \cdot \mathbf{q}_{2}\right), \\
F_{\text {cont }}^{a}(\sigma) & =+i \frac{g g_{\sigma}}{2 m^{2}} \tau_{1}^{a}\left(\boldsymbol{\sigma}_{1} \cdot \mathbf{q}_{1}\right),
\end{aligned}
$$

where (A19) can be verified with the help of $\mathbf{q}_{1}+\mathbf{q}_{\mathbf{2}}+\mathbf{q}_{\mathbf{3}}=\mathbf{0}$. The corresponding potentials are just the products of $D$ and $F$ factors, they are listed in the main body of the paper.

For the isoscalar vector meson $\omega$ only the timelike part $\mu=4$ of the vertices contribute up to the order considered

$$
\begin{aligned}
\Gamma_{\mu}(\omega, q) & =g_{\omega}\left[\gamma_{\mu}-\frac{\kappa_{\omega}}{2 m} \sigma_{\mu \nu} q_{\nu}\right] \\
D_{4}^{a}(\omega) & =\frac{g g_{\omega}}{2 m} \tau_{2}^{a}\left(\boldsymbol{\sigma}_{2} \cdot \mathbf{q}_{2}\right) \Delta_{\pi}\left(q_{2}\right) \Delta_{\omega}\left(q_{3}\right), \\
F_{\mathrm{PV}, 4}^{a,+}(\omega) & =-\frac{g g_{\omega}}{2 m^{2}} \tau_{1}^{a}\left(\boldsymbol{\sigma}_{1} \cdot \mathbf{q}_{3}\right), \\
F_{\mathrm{PS}, 4}^{a,-}(\omega) & =-\frac{g g_{\omega}\left(1+\kappa_{\omega}\right)}{2 m^{2}} \tau_{1}^{a}\left(\boldsymbol{\sigma}_{1} \cdot \mathbf{q}_{3}\right), \\
F_{\mathrm{cont}}^{a}(\omega) & =+\frac{g g_{\omega} \kappa_{\omega}}{2 m^{2}} \tau_{1}^{a}\left(\boldsymbol{\sigma}_{1} \cdot \mathbf{q}_{3}\right) .
\end{aligned}
$$


Finally, for the isovector vector meson $\rho$ the vertex function reads

$$
\Gamma_{\mu}^{b}(\rho, q)=g_{\rho}\left[\gamma_{\mu}-\frac{\kappa_{\rho}}{2 m} \sigma_{\mu \nu} q_{\nu}\right] \tau^{b}
$$

For the timelike component of the $\rho$ field $(\mu=4)$ one gets

$$
\begin{aligned}
D_{4}^{a b}(\rho) & =\frac{g g_{\rho}}{2 m} \tau_{2}^{a} \tau_{3}^{b}\left(\boldsymbol{\sigma}_{2} \cdot \mathbf{q}_{2}\right) \Delta_{\pi}\left(q_{2}\right) \Delta_{\rho}\left(q_{3}\right), \\
F_{\mathrm{PV}, 4}^{a b,+}(\rho) & =-\frac{g g_{\rho}}{2 m^{2}}\left[\delta_{a b}\left(\boldsymbol{\sigma}_{1} \cdot \mathbf{q}_{3}\right)-i \epsilon_{a b c} \tau_{1}^{c}\left(\boldsymbol{\sigma}_{1} \cdot \mathbf{Q}_{1}\right)\right], \\
F_{\mathrm{PS}, 4}^{a b,-}(\rho) & =-\frac{g g_{\rho}\left(1+\kappa_{\rho}\right)}{2 m^{2}} \delta_{a b}\left(\boldsymbol{\sigma}_{1} \cdot \mathbf{q}_{3}\right), \\
F_{\mathrm{cont}, 4}^{a b}(\rho) & =+\frac{g g_{\rho}}{2 m^{2}}\left[\delta_{a b} \kappa_{\rho}\left(\boldsymbol{\sigma}_{1} \cdot \mathbf{q}_{3}\right)+i \epsilon_{a b c} \tau_{1}^{c}\left(\boldsymbol{\sigma}_{1} \cdot \mathbf{Q}_{1}\right)\right],
\end{aligned}
$$

where $\mathbf{Q}_{\mathbf{i}}=\mathbf{p}_{\mathbf{i}}^{\prime}+\mathbf{p}_{\mathbf{i}}$. For the spacelike component

$$
\begin{aligned}
\mathbf{D}^{a b}(\rho) & =-i \frac{g g_{\rho}}{4 m^{2}} \tau_{2}^{a} \tau_{3}^{b}\left(\boldsymbol{\sigma}_{2} \cdot \mathbf{q}_{2}\right)\left[\mathbf{Q}_{3}+\left(1+\kappa_{\rho}\right) i \boldsymbol{\sigma}_{3} \times \mathbf{q}_{3}\right] \Delta_{\pi}\left(q_{2}\right) \Delta_{\rho}\left(q_{3}\right), \\
\mathbf{F}_{\mathrm{PV}}^{a b,+}(\rho) & =0 \\
\mathbf{F}_{\mathrm{PS}}^{a b,-}(\rho) & =-\mathbf{F}_{\mathrm{cont}}^{a b}(\rho)=-\frac{g g_{\rho}}{m} \epsilon_{a b c} \tau_{1}^{c} \boldsymbol{\sigma}_{1} .
\end{aligned}
$$

In the main body of the paper we denote the contributions due to the forth component of vector fields by subscript $T$ and those from the exchanges with $\mu=1,2,3$ by the subscript $S$.

\section{APPENDIX B: $\sigma$-MESON EXCHANGE IN $3 N$ POTENTIALS}

The Bonn potentials use different parameters (masses, coupling constants and cut-off parameters) of their " $\sigma$ "-meson in $N N$ channels with isospin $I=0$ and $I=1$. Let us define

$$
v\left(\sigma(I), \mathbf{q}^{2}\right)=\frac{g_{\sigma(I)}^{2}}{4 \pi} \frac{F_{\sigma(I) N N}^{2}\left(\Lambda_{\sigma(I) N N}, \mathbf{q}^{2}\right)}{m_{\sigma(I)}^{2}+\mathbf{q}^{2}},
$$

where $g_{\sigma(I)}, \Lambda_{\sigma(I) N N}, m_{\sigma(I)}^{2}$ are the Bonn $\sigma(I)$-exchange parameters in the respective $N N$ isospin channel. The dominant central part of the Bonn $\sigma$-exchange potential is then given by

$$
\begin{aligned}
v_{C}\left(\mathbf{q}^{2}\right) & =v\left(\sigma(0), \mathbf{q}^{2}\right) P_{0}+v\left(\sigma(1), \mathbf{q}^{2}\right) P_{1}, \\
P_{0} & =\frac{1}{4}\left(1+\boldsymbol{\tau}_{1} \cdot \boldsymbol{\tau}_{2}\right), \quad P_{1}=\frac{1}{4}\left(3-\boldsymbol{\tau}_{1} \cdot \boldsymbol{\tau}_{2}\right),
\end{aligned}
$$




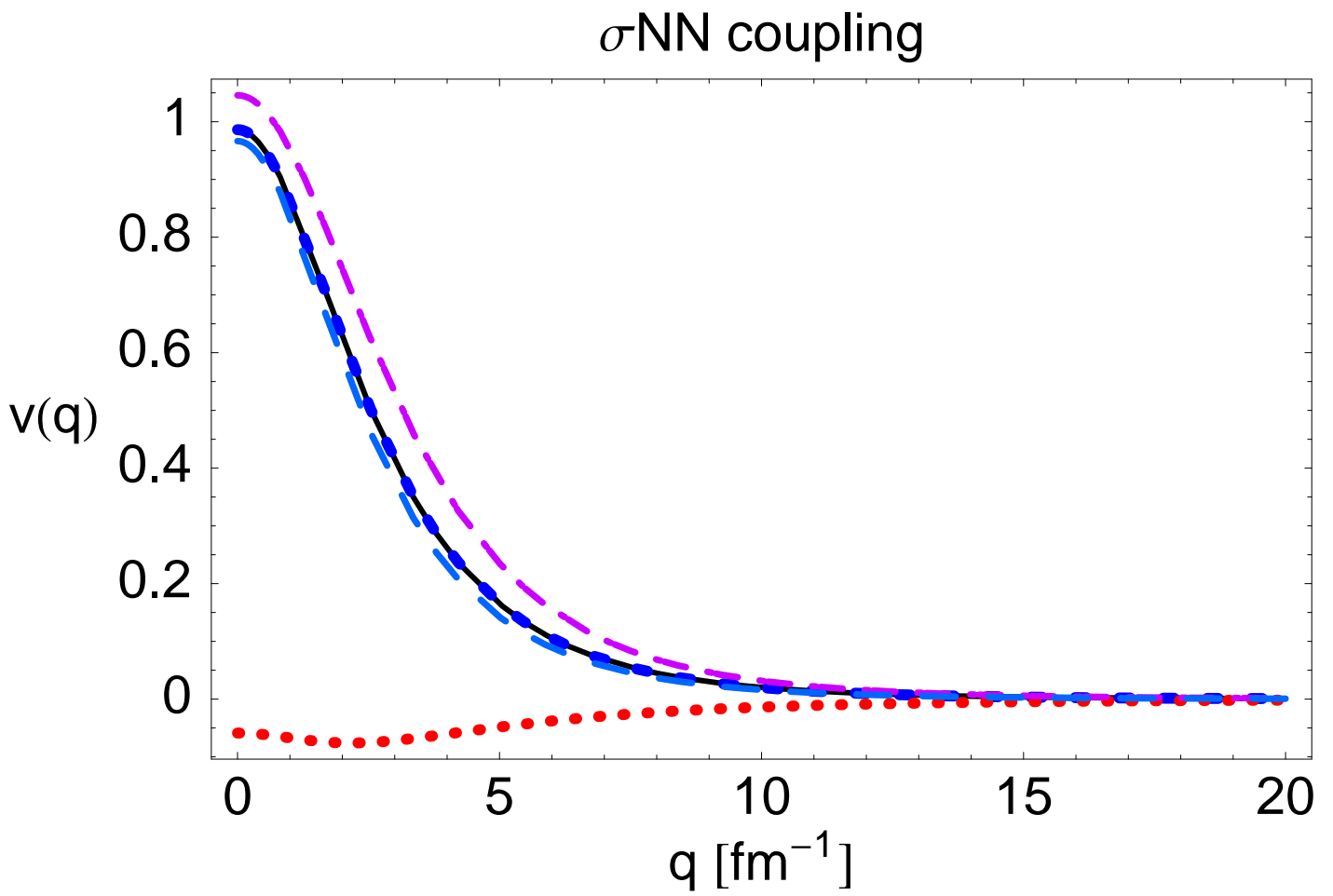

FIG. 3: The fit (solid line) to the central part of the isoscalar Bonn potential (short-dashed line; practically coincides with the solid line on the graph), the first term of Eq. (B4). For comparison, also $v\left(\sigma(0), \mathbf{q}^{2}\right)$ (dash-dotted line), $v\left(\sigma(1), \mathbf{q}^{2}\right)$ (dashed line) and their isovector combination (the second term in Eq. (B4); dotted line) are shown.

which can be re-written in a form similar to the central potentials originated from exchanges of two scalar-isoscalar and two scalar-isovector particles:

$$
v_{C}\left(\mathbf{q}^{2}\right)=\frac{1}{4}\left[v\left(\sigma(0), \mathbf{q}^{2}\right)+3 v\left(\sigma(1), \mathbf{q}^{2}\right)\right]+\boldsymbol{\tau}_{1} \cdot \boldsymbol{\tau}_{2} \frac{1}{4}\left[v\left(\sigma(0), \mathbf{q}^{2}\right)-v\left(\sigma(1), \mathbf{q}^{2}\right)\right] .
$$

This does not mean that the Bonn $\sigma$-exchange is equivalent to the true exchange of four scalar particles, since: 1) the last term has the sign opposite to the sign of a real scalar exchange; 2) all four of these exchange have to act at once, i.e., they cannot be separated by an exchange of another meson (in iterations of the Lippmann-Schwinger equation).

To have a simple prescription for the $\sigma$-exchange in our $3 N$ potentials, we fitted the first term in Eq. (B4) by the function $v\left(\sigma, \mathbf{q}^{2}\right)$ dependent on parameters of single true isoscalar $\sigma$-exchange $g_{\sigma}, \Lambda_{\sigma N N}, m_{\sigma}^{2}$. The result of the fit to the Bonn B potential gives the values listed in Table III and the quality of the fit is shown on Fig. 3. The fitted parameters are between the Bonn values for $N N I=0,1$ channels. The second ("isovector-exchange") 
component of Eq. (BB4) is much smaller in absolute value and even negative for $q \leq 10 \mathrm{fm}^{-1}$ (see Fig. 3), which precludes an approximation by the exchange of scalar particle(s). If we nevertheless include it into the $3 N$ potential, it gives a considerably smaller contribution to the triton binding energy. Therefore, we neglected it in the calculations of this paper. 
[1] R. Machleidt, Phys. Rev. C 63, 024001 (2001).

[2] V.G.J. Stocks, R.A.M. Klomp, C.P.F. Terheggen, J.J. de Swart, Phys. Rev. C 49, 2950 (1994).

[3] R. Machleidt, K. Holinde, and Ch. Elster, Phys. Rep. 149, 1 (1987).

[4] S.R. Beane, P.F. Bedaque, M.J. Savage, and U. van Kolck, Nucl. Phys. A700, 377 (2002).

[5] E. Epelbaum, W. Glöckle, Ulf-G. Meißner, Nucl. Phys. A637, 107 (1998).

[6] E. Epelbaum, W. Glöckle, Ulf-G. Meißner, Nucl. Phys. A671, 295 (2000);

E. Epelbaum, A. Nogga, W. Glöckle, H. Kamada, Ulf-G. Meißner, H. Witała, Eur. Jour. Phys. A15, 543 (2002).

[7] D.R. Entem and R. Machleidt, Phys. Rev. C 65, 064005 (2002); Phys. Rev. C 66, 014002 (2002).

[8] E. Epelbaum, Ulf-G. Meißner, W. Glöckle, Ch. Elster, Phys. Rev. C 65, 044001 (2002).

[9] D. Hüber, J.L. Friar, A. Nogga, H. Witała, U. van Kolck, Few-Body Syst. 30, 95 (2001).

[10] E. Epelbaum, A. Nogga, W. Glöckle, H. Kamada, Ulf-G. Meißner, H. Witała, Phys. Rev. C 66, 064001 (2002).

[11] S.A. Coon, M.D. Scadron, and B.R. Barrett, Nucl. Phys. A242, 467 (1975);

S.A. Coon, M.D. Scadron, P.C. McNamee, B.R. Barrett, D.W.E. Blatt, B.H.J. McKellar, Nucl. Phys. A317, 242 (1979).

[12] S.A. Coon and W. Glöckle, Phys. Rev. C 23, 1790 (1981).

[13] R.G. Ellis, S.A. Coon, and B.H.J. McKellar, Nucl. Phys. A438, 631 (1985).

[14] S.A. Coon, M. T. Peña, and D.O. Riska, Phys. Rev. C 52, 2925 (1995).

[15] J.L. Friar, D. Hüber, U. van Kolck, Phys. Rev. C 59, 53 (1999).

[16] G.E. Brown, Comments on Nucl. and Part. Phys. 4, 140 (1970);

G.E. Brown and J.W. Durso, Phys. Lett. 35B, 120 (1971).

[17] H.T. Coelho, T.K. Das, and M.R. Robilotta, Phys. Rev. C 28, 1812 (1983).

[18] S. Weinberg, Physica 96A, 327 (1979).

[19] U. van Kolck, Phys. Rev. C 49, 2932 (1994);

C. Ordonêz, L. Ray, and U. van Kolck, Phys. Rev. C 53 (1996) 2086.

[20] S.A. Coon, H.K. Han, Few Body Syst. 30, 131 (2001).

[21] D. Hüber and J.L. Friar, Phys. Rev. C 58, 674 (1998). 
[22] A. Kievsky, Phys. Rev. C 60, 034001 (1999).

[23] E. Epelbaum, H. Kamada, A. Nogga, H. Witała, W. Glöckle, Ulf-G. Meißner, Phys. Rev. Lett. 86, 4787 (2001).

[24] L. Canton, W. Schadow, Phys. Rev. C 62, 044005 (2002);

L. Canton, W. Schadow, J. Haidenbauer, Eur. Phys. J. A14, 225 (2002).

[25] S.C. Pieper, V.R. Pandharipande, R.B. Wiringa, J. Carlson, Phys. Rev. C 64, 014001 (2001).

[26] B.S. Pudliner, V.R. Pandharipande, J. Carlson, S.C. Pieper, and R.B. Wiringa, Phys. Rev. C 56, 1720 (1997).

[27] H. Heiselberg, V. Pandharipande, Ann. Rev. Nucl. Part. Sci. 50, 481 (2000).

[28] S.A. Coon, W. Glöckle, Phys. Rev. C 23, 1796 (1981);

S.A. Coon, J.L. Friar, Phys.Rev. C 34, 1060 (1986).

[29] S.A. Coon, M.T. Peña, Phys. Rev. C 48, 2559 (1993).

[30] A. Stadler, J. Adam Jr. , H. Henning, and P. U. Sauer, Phys. Rev. C 51, 2896 (1995).

[31] J. Smejkal, E. Truhlík, H. Göller, Nucl. Phys. A624, 655 (1997), and references therein.

[32] M. Soyeur, Nucl. Phys. A671, 532 (2000).

[33] S. Hirenzaki, P. Fernández de Córdoba, E. Oset, Phys. Rev. C 53, 277 (1996).

[34] A. Stadler, W. Glöckle, and P.U. Sauer, Phys. Rev. C 44, 2319 (1991).

[35] J. Adam, Jr., "Proceedings of XIVth International Conference on Few Body Problems in Physics", May 26-31, 1994, Williamsburg, Virginia edited by Franz Gross, AIP 334, 192 (1994).

[36] M. Chemtob, M. Rho, Nucl. Phys. A163, 1 (1971);

D.O. Riska, Prog. Part. Nucl. Phys. 11, 199 (1984).

[37] J. Adam, Jr., E. Truhlík, D. Adamová, Nucl. Phys. A492, 556, (1989).

[38] R. V. Reid, Ann. Phys. (N.Y.) 50, 411 (1968).

[39] M. Lacombe, B. Loiseau, J. M. Richard, R. Vinh Mau, J. Côté, P. Pirès, and R. de Tourreil, Phys. Rev. C 21, 861 (1980).

[40] R. Machleidt, Adv. Nucl. Phys. 19, 189 (1989). 